Tunable nano-wrinkling of chiral surfaces: Structure and diffraction optics

P. Rofouie, D. Pasini, and A. D. Rey'

Citation: The Journal of Chemical Physics 143, 114701 (2015); doi: 10.1063/1.4929337

View online: http://dx.doi.org/10.1063/1.4929337

View Table of Contents: http://aip.scitation.org/toc/jcp/143/11

Published by the American Institute of Physics

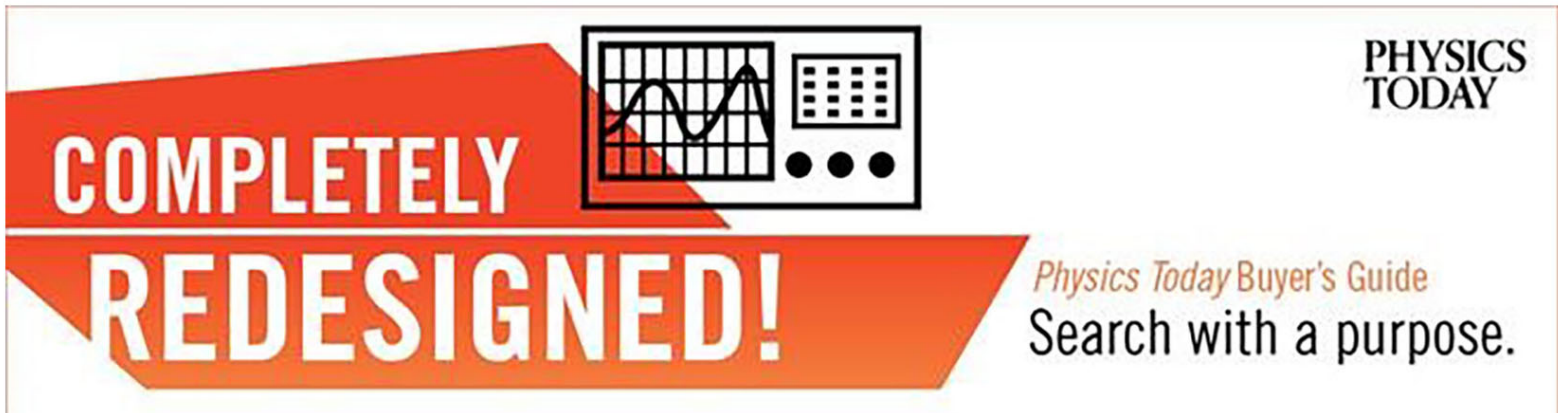




\title{
Tunable nano-wrinkling of chiral surfaces: Structure and diffraction optics
}

\author{
P. Rofouie, ${ }^{1}$ D. Pasini, ${ }^{2}$ and A. D. Rey ${ }^{1, a)}$ \\ ${ }^{1}$ Department of Chemical Engineering, McGill University, 3610 University Street, Montreal, \\ Quebec H3A 2B2, Canada \\ ${ }^{2}$ Department of Mechanical Engineering, McGill University, 817 Sherbrook West, Montreal, \\ Quebec H3A OC3, Canada
}

(Received 26 May 2015; accepted 24 July 2015; published online 15 September 2015)

\begin{abstract}
Periodic surface nano-wrinkling is found throughout biological liquid crystalline materials, such as collagen films, spider silk gland ducts, exoskeleton of beetles, and flower petals. These surface ultrastructures are responsible for structural colors observed in some beetles and plants that can dynamically respond to external conditions, such as humidity and temperature. In this paper, the formation of the surface undulations is investigated through the interaction of anisotropic interfacial tension, swelling through hydration, and capillarity at free surfaces. Focusing on the cellulosic cholesteric liquid crystal (CCLC) material model, the generalized shape equation for anisotropic interfaces using the Cahn-Hoffman capillarity vector and the Rapini-Papoular anchoring energy are applied to analyze periodic nano-wrinkling in plant-based plywood free surfaces with water-induced cholesteric pitch gradients. Scaling is used to derive the explicit relations between the undulations' amplitude expressed as a function of the anchoring strength and the spatially varying pitch. The optical responses of the periodic nano-structured surfaces are studied through finite difference time domain simulations indicating that CCLC surfaces with spatially varying pitch reflect light in a wavelength higher than that of a CCLC's surface with constant pitch. This structural color change is controlled by the pitch gradient through hydration. All these findings provide a foundation to understand structural color phenomena in nature and for the design of optical sensor devices. (C) 2015 AIP Publishing LLC. [http://dx.doi.org/10.1063/1.4929337]
\end{abstract}

\section{INTRODUCTION}

A variety of surface ultrastructures can be found in many biological materials, such as the periodic undulations observed in films of concentrated collagen, ${ }^{1}$ silk gland ducts of the golden orb-web spider Nephila clavipes, ${ }^{2}$ the exoskeleton of the beetle Chrysina gloriosa, ${ }^{3}$ and other cellulosic materials. ${ }^{4,5}$ It has been shown that the surface patterns in the exoskeleton of Chrysina gloriosa and in the duct of silk secreting glands in Nephila clavipes closely resemble the atomic force microscopy image of the focal conic domains that spontaneously arise on the free surface of cholesteric liquid crystals, CLCs. ${ }^{3,6}$ Besides, similar periodic patterns have been observed at the free surface of a nematic liquid crustal under a magnetic field ${ }^{7}$ and a cholesteric under a homeotropic alignment. ${ }^{8}$ Cholesteric liquid crystal self-assembly has been proposed to explain the surface structures of many fibrous composites ranging from plant cell walls to arthropod cuticles. ${ }^{9}{ }^{910}$ Some of these periodic surface structures give rise to structural colors that are observed in beetles, ${ }^{3}$ mollusk shells, ${ }^{11}$ ostracods,${ }^{12}$ large fishes,${ }^{13}$ and some plants. ${ }^{14}$ Although, minor light absorption effects have been reported in CLCs, the absorption of light by pigments in some beetle cuticles plays an important role in structural colors. The black melanin pigment present in a Japanese jewel beetle cuticle absorbs the light fraction scattered from inner layers, which contributes in determining the iridescent color. ${ }^{15}$

\footnotetext{
a)Electronic mail: alejandro.rey@mail.mcgill.ca
}

Furthermore, the single iridescent colors observed in some beetles and butterflies result from color mixing of structurally different colors. The beetle Calidea panaethiopica shows a metallic green color that originates from a multilayer structure generating two different colors mixed to produce the single color. ${ }^{16}$ Additionally, the complex green color of the butterfly Papilio palinurus results from an additive color mixing of yellow and blue. ${ }^{17}$

Structural color and iridescence in plants are typically associated with diffraction grating, thin layered or multilayered interference, and often with contributions from pigmentation as well. Structural color in plant's leaves arises essentially from multilayers of cellulose microfibrils with differing orientations in the plant cell walls forming a cholesteric liquid crystal phase. ${ }^{18,19}$ Structural color in plant's flowers arises essentially from surface diffraction gratings. For example, the petals of Hibiscus trionum and Tulipa kaufmanniana feature ordered surface striation controlling iridescence with a striking metallic appearance. ${ }^{20}$

Color change in response to environmental stimuli is ubiquitous in plants and animals. The iridescent blue color in the fish Chrysiptera cyanea can change to ultraviolet by the simultaneous change in spacing of the adjoining reflecting plates. ${ }^{21}$ It has also been observed that the beetles Dynastes hercules and Coptocycla can vary their coloration in response to humidity. ${ }^{22,23}$ The elytra of longhorn beetles Tmesisternus isabellae reacts to water absorption by color change, from golden in the dry state to red in the wet state. The factors 
controlling color change are the swelling of the multilayer pitch (from about $175 \mathrm{~nm}$ in the dry state to about $190 \mathrm{~nm}$ in the fully wet state) and the water infiltration. ${ }^{24}$ Structural colors in some plants can also be the result of a response to humidity, light, and touch through the change of bulk and surface nanostructures, among other external stimuli. One of the interesting examples is a tropical rainforest plant, Selaginella willdenowii that possesses iridescent blue leaves that can dynamically respond to hydration and dehydration. It has been shown that the iridescent blue color turns to green when the leaves are immersed in water, with blue color reappearing when the leaves are dry. ${ }^{25}$ Electron microscopy of iridescent blue leaves in four Malaysian rain forest understory plants shows that optical effects upon humidity changes are mediated by altering the twisted plywood structure (helix pitch) in the plant cell walls. The reported lamellar spacing in green leaves is 268 $\pm 2.2 \mathrm{~nm}$, which is larger than the lamellar spacing in blue leaves, $141 \pm 2.4 \mathrm{~nm} .{ }^{18}$ Similar behavior is observed in a hygroscopic liquid crystal polymer film that responds to temperature and humidity by changing its color. ${ }^{26}$ It has been proved that that when humidity is high, the film swelling results in a pitch increase as opposed to the case of low humidity, which causes a decrease in the pitch with consequent film shrinkage.

Understanding the mechanisms through which plants exhibit specific optical appearances is an illustration of "bioinspiration" for the design of optical devices with novel functionalities ${ }^{27}$ and as a tool to characterize plant-based plywoods. Although the formation of these surface ultrastructures during the plant development is not yet well understood, it is recognized that the formation and periodicity of the plant surface undulations are governed by the cellulosic cholesteric liquid crystal (CCLC) structure. ${ }^{28}$ It has been shown that the change of the orientational order, the fiber orientation at the CCLC interface, defined by the average direction of preferred orientation called the director $\mathbf{n}$, and the presence of topological defects can cause the undulation on free interfaces through a process of surface energy minimization of the surface interface shape ${ }^{29}$ The interaction between the interface shape and the director $\mathbf{n}$ is a complex phenomenon involving interfacial tension, surface anchoring energy, and LC Frank elasticity, all factors requiring the integration of multi-scale modelling for bulk and surface structure properties. ${ }^{30}$ In our recent study, we presented the main mechanisms driving nanoscale surface undulations in chiral nematic liquid crystals as shown in plantbased plywoods and various cholesteric liquid crystals. ${ }^{31,32}$

Of particular interest in the present paper is the study the role of tangential water gradient in the formation of plant surface undulations. The insight gained from this study can be used to generate surface patterns and nano-scale actuation systems capable to autonomously respond to humidity. In this paper, we seek to characterize the free surface relief of CCLC through the interaction of anisotropic interfacial tension, swelling through hydration, and capillarity at free surfaces. With a focus on the CCLC material model, we consider twodimensional cholesteric films with a free interface subject to a tangential water gradient. To analyze periodic nanowrinkling in plant-based plywood free surfaces, we use the generalized shape equation for anisotropic interfaces using the Cahn-Hoffman capillarity vector developed for LCs, ${ }^{33}$ and the classical Rapini-Papoular anchoring energy ${ }^{34}$ for the anisotropic part of the interfacial tension. To investigate the optical responses of the surface wrinkling, we implement finite difference simulations in the time domain (FDTD) and calculate the corresponding planar reflection of light. The FDTD method is established as an accurate numerical method to solve Maxwell's equations and as an efficient tool for simulation of light propagation in liquid crystals containing complex textures. ${ }^{35}$ It has been shown that the FDTD method is capable of predicting optical responses to textured nematic liquid crystal films containing non-uniform orientation induced by variations in surface anchoring. ${ }^{36}$ The interaction between the incident light and the nanoscale periodic structure shows bistructural color reflection through hydration and dehydration. The wavelength of the reflection peak strongly depends on the CCLC helical pitch. The self-assembly formation of the CLC surface ultrastructure together with the water-induced multiple structural colors suggests a potential mechanism to exploit in the design of colorimetric humidity sensors.

The organization of this paper is as follows. Section II introduces the CCLCs material model system with waterinduced spatially varying pitch, the interfacial force balance equation, and the governing nemato-capillary shape equation expressing the coupling mechanism between the surface geometry and cellulose fiber orientation for CCLC/air interface in rectangular $(\mathrm{x}, \mathrm{y}, \mathrm{z})$ coordinates. The capillary shape equation is derived and the unique role of the Cahn-Hoffman capillary vector is discussed. Section III presents the effect of model parameters on the surface profile, the capillary pressures associated with the CCLC interface, the scaling formula expressing surface profile amplitude as a function of the anchoring strength and the water-induced varying helix pitch, and the optical response of the surface relief using the finite difference time domain simulation. In Section III, the reflection of light affected by the spatially varying pitch is also theoretically investigated and compared with the reflection of light at free surfaces of CCLCs with constant pitch. The role of key parameters, such as the anchoring strength, and the helix pitch on the optical properties is discussed. Section IV presents conclusions.

\section{THEORY AND GOVERNING EQUATIONS}

\section{A. Geometry and structure}

Plant cell walls can be considered as a natural viscoelastic composite reinforced by cellulose microfibrils (CMFs) coated with hemicelluloses and embedded in a matrix of lignin/pectin. ${ }^{37} \mathrm{CMFs}$ in the extracellular polysaccharide matrix are oriented in strategic directions to form the twisted plywood architecture that provides mechanical strength, controls cell expansion, contributes to the morphology at the tissue, and functions in signaling. ${ }^{38} \mathrm{~A}$ plant cell wall consists of a primary wall that is a thin layer laid down during cell growth, and three secondary walls, which are thick and rigid layers, laid down when the cell has reached its final size and shape. ${ }^{38}$ However, the mechanisms by which the CMFs are oriented in a specific direction are not well understood. Based on the microstructural and textural similarities between the plant cell 


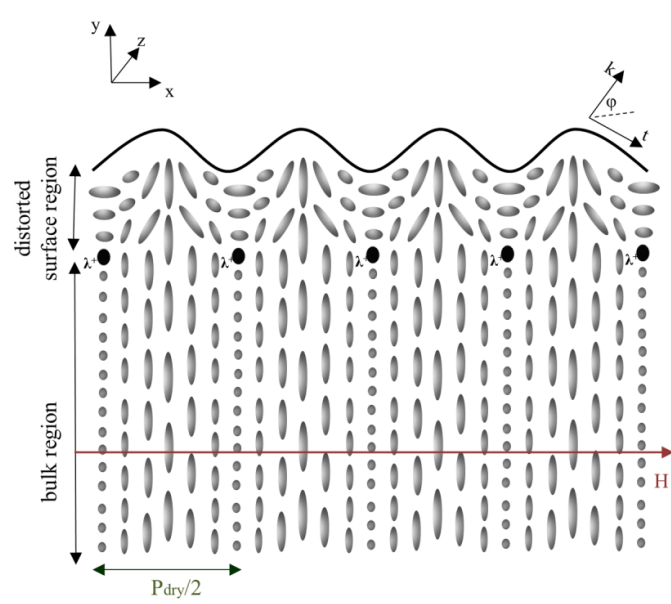

(a)

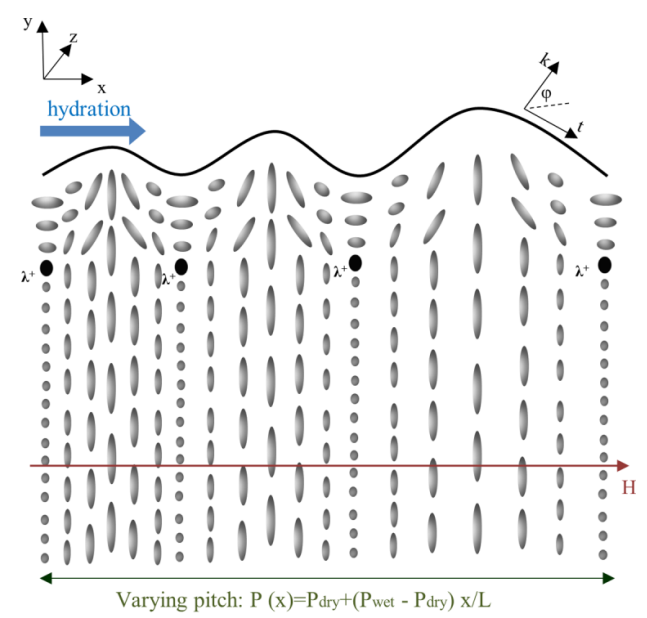

(b)

FIG. 1. Schematic of a cholesteric liquid crystal (plywood architecture) and nano-wrinkled surface structures. Bend and splay orientation distortions with $\lambda^{+}$ disclination for normal anchoring $(\mathrm{W}<0)$ create surface undulations. Note that the director field is continuous everywhere and the $\lambda^{+}$lines have a low energy non-singular core. (a) Surface structure of CCLC surface with constant pitch. $\mathbf{H}$ is the helix unit vector, and $\mathrm{P}_{\text {dry }}$ is the constant pitch. (b) Surface structure of CCLC surface with water-induced varying pitch. $\mathrm{P}(\mathrm{x})$ is the local pitch which varies linearly over a range of $\mathrm{x}$-values: $\mathrm{P}(\mathrm{x})=\mathrm{P}_{\mathrm{dry}}+\left(\mathrm{P}_{\mathrm{wet}}-\mathrm{P}_{\mathrm{dry}}\right) \mathrm{x} / \mathrm{L}$. Adapted with permission from Meister et al., J. Phys. II 6(6), 827-844 (1996). Copyright 1996 EDP Sciences.

walls and chiral liquid crystal phases, it is hypothesized that the formation of plant cell wall microstructure arises through liquid crystal self-assembly. ${ }^{39-41}$ Plant cell walls can be characterized by a multi-layered structure where cellulose fibers are laid down parallel in each layer, whereas the CMF orientation between layers changes by a constant angle. ${ }^{10} \mathrm{Fig}$. 1(a) depicts the schematics of the helicoidal plant cell wall where ellipsoids indicate fiber orientation on each parallel layer. The fiber orientation at the interface is defined by the director $\mathbf{n}$. The instantaneous axis of rotation is defined by the helix axis $\mathbf{H}$. We assume that the helix axis remains parallel to the surface; other complex structures occurring when the helix axis $\mathbf{H}$ is distorted are beyond the scope of this paper. The pitch length $P$ is defined as the distance through which the fibers undergo a $2 \pi$ rotation. The handedness of the CLC in the whole study is assumed to be right-handed $(\mathrm{P}>0)$. For rectangular $(\mathrm{x}, \mathrm{y}, \mathrm{z})$ coordinate system, the surface relief that is directed along the $\mathrm{x}$ axis can be described by a $y(x, z)$ deviation from the $x z$ plane. The amplitude of the vertical undulation is $h(x)$. As the surface relief is constant in the $\mathrm{z}$ direction for a linear texture, the curvature in the $\mathbf{z}$-direction is zero. The unit tangent, $\mathbf{t}$ and the unit normal, $\mathbf{k}$ to the surface can be expressed with the normal angle, $\varphi:(\mathbf{x})$ $=(\sin \varphi(\mathrm{x}),-\cos \varphi(\mathrm{x}), 0), \mathbf{k}(\mathrm{x})=(\cos \varphi(\mathrm{x}), \sin \varphi(\mathrm{x}), 0)$. $\mathrm{L}$ is the given system length in the $\mathrm{x}$ direction. The arc-length measure of the undulating surface is "s."

The preferred fiber orientation or easy axis at the interface can be parallel to the unit normal $\mathbf{k}$ (homeotropic) or perpendicular to the unit normal $\mathbf{k}$ (planar). Based on the surface anchoring type (planar or homeotropic) of CLCs, the director filed configuration close to the surface can be distorted or undistorted to reach the minimum-energy state. In the case of planar anchoring, as the helix axis $\mathbf{H}$ perpendicular to the surface is the most stable configuration, it will remain undistorted. ${ }^{42}$ However, in the case of homeotropic anchoring $(\mathrm{W}<0)$, either the helix structures parallel to the surface or perpendicular to the surface is not fully compatible with any uniform aligning surface. ${ }^{43}$ Therefore, to adapt the helix axis to uniformly aligning surface, a set of elastic distortion and sub-surface defect nucleation results in a disruption of the average orientational order and creation of topological defects, need to be formed at the interface. In this paper, we restrict the discussion to homeotropic anchoring ( $\mathrm{W}<0$ ), because the undistorted helicoidal structure for planar surface anchoring (W $>0, \mathbf{n} \cdot \mathbf{k}=0$ ) results in a flat surface. ${ }^{6}$ It has been reported that the presence of elastic distortion near to the CLC interface can change the director field and its periodicity at the free surface. ${ }^{44,45}$ Here, we assume that the periodicity of the distorted region is constant and equal to the bulk pitch, P. Usually, the defect dissociates in a bulk $\lambda^{+}$and a surface $\tau^{-}$ pair. The $\tau^{-}$defect lines disappear for the non-planar surface (see Fig. 1(a)).

The present paper uses the cholesteric representation of the director field $\mathbf{n}$ developed by Meister et al., ${ }^{6}$

$$
\begin{gathered}
\mathbf{n}(\mathrm{x}, \mathrm{y})=(\cos \mathrm{qx} \cos \mathrm{qy}, \sin \mathrm{qx}, \cos \mathrm{qx} \sin \mathrm{qy}), \\
\mathrm{q}(\mathrm{x})=\frac{2 \pi}{\mathrm{P}(\mathrm{x})} .
\end{gathered}
$$

Topological defects in cholesteric liquid crystals include disclinations and dislocations. Disclination is a defect involving discontinuity in the director field, and dislocation is a translational defect corresponding to addition or removal of cholesteric layers. Disclinations are characterized by core type (singular, $\tau$ lines or non-singular, $\lambda$ lines), where in singular core the molecular order deviates from its homogeneous value while in the non-singular core it does not. The strength of disclination is characterized by sense rotation (sign) and amount of rotation (amplitude). ${ }^{46}$

We note that this director field is continuous everywhere and only contains non-singular core $\lambda^{+}$disclination lines, and that $\mathrm{n}_{y}(\mathrm{x})=\sin q x$ lacks " $\mathrm{y}$ " dependence. The latter is an important factor for the bulk normal stresses. The interfacial structure, with an array of $\lambda^{+}$lines at a distance $\mathrm{P} / 4$ from the surface, emerges from a sufficiently strong homeotropic anchoring. Fig. 2(a) shows the director $\mathbf{n}$ trajectories on the 


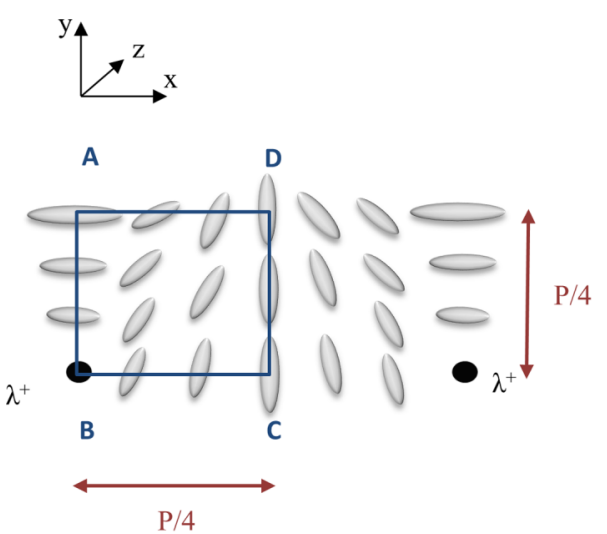

(a)

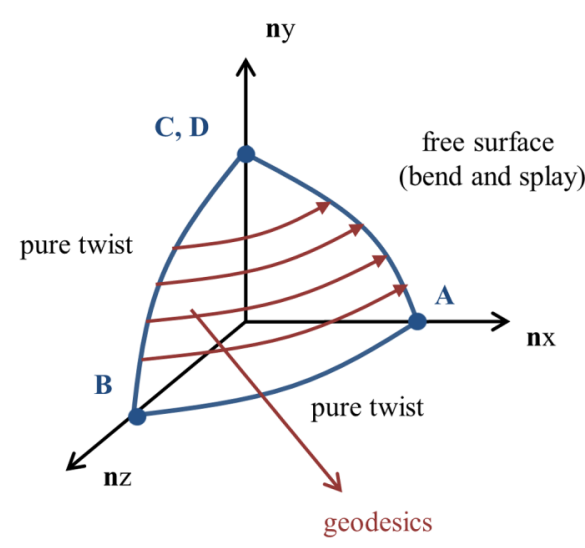

(b)

FIG. 2. (a) The director field used in this paper is continuous everywhere in ABCD. Adapted with permission from Meister et al., J. Phys. II 6(6), 827-844 (1996). Copyright 1996 EDP Sciences. (b) The corresponding orientation paths $\left(\mathrm{n}_{\mathrm{y}}(\mathrm{x})\right.$ ) are geodesics, well-known energy minimizers. ${ }^{47,48}$

unit sphere corresponding to Figure 1. It has been proved that for equal elastic constants, all the trajectories on the unit sphere are geodesic, i.e., circles whose plane passes through the center of the sphere. Geodesics are well-known energy minimizers for liquid crystals. ${ }^{47,48}$ As depicted in Fig. 2(b), the continuous director field is the ABCD patch on the unit sphere limited by two meridians (pure twist A-B and splaybend A-D) and the equator. The temperature and concentration dependence of the CLC helical pitch has been widely reported. ${ }^{7}$ Recently Matsuyama ${ }^{49,50}$ showed that the helical pitch of a cholesteric phase is a function of the concentration and the orientation order parameter in a mixture of polymer and a liquid crystal. In the present study, we assume that the helix pitch changes in response to humidity level from the swelling and shrinking of the polysaccharide matrix through water sorption. As the stiffness of the crystalline cellulose microfibrils is approximately two orders of magnitude higher than the hygroscopic matrix, they do not swell whereas the hygroscopic matrix, which is embedded in, does. ${ }^{51}$

It has been shown that some biological helicoidal structures when exposed to higher humidity result in an increase of the pitch due to the swelling of the multilayer, and when exposed to lower humidity, they result in a decrease of the pitch due to the shrinkage of the multilayer. Therefore, in the presence of the tangential water gradient at the CLC-air interface, we consider a spatially varying pitch, where the director $\mathbf{n}$ rotates non-uniformly along the $\mathrm{x}$-direction. The pitch gradient can be described by a power series or an exponential. ${ }^{52,53} \mathrm{To}$ meet the objectives and remain within the scope of this paper, we assume the pitch profile as linear. As shown in Fig. 1(b), in the presence of the tangential water gradient, the pitch is assumed to grow from $\mathrm{P}_{\text {dry }}$ to $\mathrm{P}_{\text {wet }}$ along the $\mathrm{x}$-direction,

$$
\mathrm{P}(\mathrm{x})=\mathrm{P}_{\mathrm{dry}}+\left(\mathrm{P}_{\mathrm{dry}}-\mathrm{P}_{\mathrm{wet}}\right) \frac{\mathrm{x}}{\mathrm{L}} .
$$

\section{B. Governing equations}

The formation of the surface undulations in CLC interfaces is a complex multiple-coupling problem that includes surface tension, anchoring energy, and bulk elasticity. To explore the free surface relief, the total system energy including bulk surface energy and the bulk elastic energy should be minimized. All the equations that govern the shape of the interfaces coupling bulk and surface phases have been presented in Ref. 30. However, the analytic solution of the problem with the usual formalism is very complicated. In the present study, to elucidate the bulk contribution to the shape equation, the interfacial stress boundary conditions at the CLC/air interface are considered. The surface undulations in plant cell walls are assumed to be formed through a modulation in the surface energy at the anisotropic-air interface.

The macroscopic chirality of the cellulose fibers emerges as the property that impacts the interfacial tension and stimulates the free surface relief. In this section, the coupling mechanism between the surface geometry and cellulose fiber orientation is presented through the shape equation based on the vector formalism of Cahn-Hoffman capillarity ${ }^{54}$ for a cholesteric liquid crystal with a spatially varying pitch (Eq. (2)).

\section{Cahn-Hoffman capillarity vector}

Cahn and Hoffman formulated the capillary vector $\xi^{54}$ to describe interfacial surface energy anisotropy and capture the changes in interfacial energy not only due to changes in interface area (dilation mode) but also due to interface tilting. This formulation was later extended and applied to anisotropic soft matter materials including liquid crystal free surfaces, and their interfaces with soft solids. For transversely isotropic materials, the orientation-dependent surface energy can be specified by the unit vector or director $\mathbf{n}=\mathbf{n}(\mathbf{r})$ field, the surface position vector $\mathbf{r}=\mathbf{r k}$, and the surface unit normal k. Cahn and Hoffman defined the nematic capillarity by the gradient of the scalar field $\mathrm{r} \gamma,{ }^{55}$

$$
\xi(\mathbf{n}, \mathbf{k})=\nabla[\mathrm{r} \gamma(\mathbf{k})] .
$$

For isotropic interfaces, the capillary vector $\xi$ reduces to a normal vector $\xi=\gamma \mathbf{k}$. The decomposition of the surface director field into normal and tangential components yields $\mathbf{n}_{\perp}=\mathbf{k k} \cdot \mathbf{n}$ and $\mathbf{n}_{\|}=\mathbf{I}_{\mathbf{s}} \cdot \mathbf{n}$, where $\mathbf{I}_{\mathrm{s}}$ is the $2 \times 2$ unit surface dyadic $\mathbf{I}_{\mathrm{s}}=\mathbf{I}-\mathbf{k k}$, and $\mathbf{I}$ is the $3 \times 3$ unit tensor. Calculating 
the gradient of the field $\mathrm{r} \gamma$ appearing in Eq. (3), using $\boldsymbol{\xi} \cdot \mathbf{k}=\gamma$ $(\mathbf{k}, \mathbf{n}), \boldsymbol{\xi} \cdot \mathrm{d} \mathbf{k}=\mathrm{d} \gamma(\mathbf{k}, \mathbf{n})$, and $\mathrm{d}(\mathrm{r} \gamma)=\nabla(\mathrm{r} \gamma) \cdot \mathrm{d} \mathbf{r}$ gives

$$
\xi(\mathbf{n}, \mathbf{k})=\nabla[\mathrm{r} \gamma(\mathbf{k})]=\gamma \frac{\partial \mathrm{r}}{\partial \mathbf{r}}+r \frac{\mathrm{d} \gamma}{\mathrm{d} \mathbf{r}}=\underbrace{\gamma \mathbf{k}}_{\xi_{\perp}}+\underbrace{\mathrm{I}_{\mathrm{s}} \cdot \frac{\mathrm{d} \gamma}{\mathrm{d} \mathbf{k}}}_{\xi_{\|}},
$$

where the normal vector component $\boldsymbol{\xi}_{\perp}$ represents the change in surface energy by contraction of the surface area, and the tangential component $\xi_{\|}$represents the change in surface energy by rotation of the surface unit normal. The classical Rapini and Papoular form of surface free energy of liquid crystals can be used to derive the Cahn-Hoffman capillary vector $\xi$ for cholesteric surfaces, ${ }^{34}$

$$
\gamma(\mathbf{n}, \mathbf{k})=\gamma_{0}+\gamma_{\text {aniso }}(\mathbf{n}, \mathbf{k}) ; \quad \gamma_{\text {aniso }}(\mathbf{n}, \mathbf{k})=\frac{\mathrm{W}}{2}(\mathbf{n} \cdot \mathbf{k})^{2},
$$

where $\gamma_{0}$ is the isotropic surface tension, $\gamma_{\text {aniso }}$ represents the anisotropic anchoring energy contribution due to the director field, and $\mathrm{W}$ is the surface anchoring strength. For strong anchoring, the director field is fixed as a function of temperature and does not change due to bulk distortions or external fields. However, for weak anchoring, the director field can be changed due to bulk director gradients and/or external fields. As mentioned above, $\mathrm{W}>0$, the easy axis (energetically preferable direction for the surface orientation) is tangential to the interface (planar), and if $\mathrm{W}<0$, the easy axis is normal to the interface (so-called homeotropic anchoring). Substituting Eq. (5) into Eq. (4) yields the following normal and tangential components of the capillarity vector:

$$
\begin{gathered}
\boldsymbol{\xi}_{\perp}(\mathbf{n}, \mathbf{k})=\left(\gamma_{0}+\frac{\mathrm{W}}{2}(\mathbf{n} \cdot \mathbf{k})^{2}\right) \mathbf{k}, \\
\boldsymbol{\xi}_{\|}(\mathbf{n}, \mathbf{k})=\mathrm{W}(\mathbf{n} \cdot \mathbf{k}) \mathbf{n}-\mathrm{W}(\mathbf{n} \cdot \mathbf{k})^{2} \mathbf{k}=\mathrm{W}(\mathbf{n} \cdot \mathbf{k})(\mathbf{n}-(\mathbf{n} \cdot \mathbf{k}) \mathbf{k}) .
\end{gathered}
$$

\section{Interfacial force balance equation}

In this section, we present the interfacial stress boundary conditions at the interface between air and cholesteric liquid crystal, denoted by phase (a) and phase (b), respectively. We assume that the system is isothermal, both phases are incompressible, and the interface is elastic. The interfacial force balance equation is the balance between interfacial forces and the bulk stress jump (SJ), ${ }^{56}$

$$
-\mathbf{k} \cdot\left(\mathbf{T}^{\mathrm{a}}-\mathbf{T}^{\mathrm{b}}\right)=\left(\nabla_{\mathrm{s}} \cdot \mathbf{T}_{\mathrm{s}}\right),
$$

where $\nabla_{\mathrm{S}}=\mathrm{I}_{\mathrm{s}} \cdot \nabla$ is the surface gradient. The surface stress tensor, $\mathbf{T}_{\mathrm{s}}$, is given by the sum of normal $\mathbf{T}^{\mathrm{N}}$, bending $\mathbf{T}^{\mathrm{B}}$, and distortion stresses $\mathbf{T}^{\mathrm{D}}$,

$$
\begin{gathered}
\mathbf{T}_{\mathrm{s}}=\mathbf{T}^{\mathrm{N}}+\mathbf{T}^{\mathrm{B}}+\mathbf{T}^{\mathrm{D}} ; \mathbf{T}^{\mathrm{N}}=\gamma \mathbf{I}_{\mathrm{s}}, \\
\mathbf{T}^{\mathrm{B}}=-\mathbf{I}_{\mathrm{s}} \cdot \frac{\partial \gamma}{\partial \mathbf{k}} \mathbf{k}, \mathbf{T}^{\mathrm{D}}=-\mathbf{I}_{\mathrm{s}} \cdot \frac{\partial \gamma}{\partial \nabla_{\mathrm{s}} \mathbf{n}} \cdot\left(\nabla_{\mathrm{s}} \mathbf{n}\right)^{\mathrm{T}},
\end{gathered}
$$

$\mathbf{T}^{\mathrm{a}}$ and $\mathbf{T}^{\mathrm{b}}$ is the total stress tensor in the air and cholesteric bulk phase, respectively. The bulk stress tensor in air is pure pressure,

$$
\mathbf{T}^{\mathrm{a}}=-\mathrm{p}^{\mathrm{a}} \mathbf{I} .
$$

The bulk stress tensor in CLC is given by ${ }^{56}$

$$
\mathbf{T}^{\mathrm{b}}=-\left(\mathrm{p}^{\mathrm{b}}-\mathrm{f}_{\mathrm{b}}\right) \mathbf{I}+\mathbf{T}^{\mathrm{E}}, \quad \mathbf{T}^{\mathrm{E}}=-\frac{\partial \mathrm{f}_{\mathrm{b}}}{\partial \nabla \mathbf{n}} \cdot(\nabla \mathbf{n})^{\mathrm{T}},
$$

where $\mathrm{p}^{\mathrm{b}}$ is the hydrostatic pressure, $\mathbf{T}^{\mathrm{E}}$ is the Ericksen stress tensor, $\mathrm{f}_{\mathrm{b}}$ is the CLC bulk Frank energy density. Substituting Eqs. (10) and (11) into Eq. (8) and projecting the result along $\mathbf{k}$ (normal component) result in

$$
\underbrace{\left(\mathrm{p}^{\mathrm{a}}-\mathrm{p}^{\mathrm{b}}\right)+\left(\mathrm{f}_{\mathrm{b}}\right)-\mathbf{k k}:\left(\frac{\partial \mathrm{f}_{\mathrm{b}}}{\partial \nabla \mathbf{n}} \cdot(\nabla \mathbf{n})^{\mathrm{T}}\right)}_{\text {bulk normal stress jump, SJ }}=\underbrace{\left(\nabla_{\mathrm{s}} \cdot \mathbf{T}_{\mathrm{s}}\right) \cdot \mathbf{k}}_{\text {-capillary pressure, }-\mathrm{p}_{\mathrm{c}}} .
$$

Simply by considering the semi-infinite media in the vertical direction and periodic in the horizontal direction neglecting gravity, the hydrostatic term, $\left(\mathrm{p}^{\mathrm{a}}-\mathrm{p}^{\mathrm{b}}\right)$, becomes zero. The remaining contribution to $\mathrm{SJ},\left\{f_{b}+\mathbf{k k}: \mathbf{T}^{\mathrm{E}}\right\}$, is known as the elastic correction in the liquid crystal literature. The expression for the bulk elastic energy density of a cholesteric is

$$
\begin{aligned}
\mathrm{f}_{\mathrm{b}}= & \frac{1}{2} \mathrm{~K}_{1}(\nabla \cdot \mathbf{n})^{2}+\frac{1}{2} \mathrm{~K}_{2}(\mathbf{n} \cdot \nabla \times \mathbf{n}-\mathrm{q}(\mathrm{x}))^{2} \\
& +\frac{1}{2} \mathrm{~K}_{3}(\mathbf{n} \times \nabla \times \mathbf{n})^{2},
\end{aligned}
$$

where $K_{1}, K_{2}$, and $K_{3}$ are the splay, twist, and bend Frank constants. $\mathrm{q}(\mathrm{x})$ is the variable wave vector equals to $2 \pi / \mathrm{P}(\mathrm{x})$. Considering one constant approximation for the Frank bulk energy and the director field expressed by Eq. (1), the corresponding Frank energy density $\mathrm{f}_{\mathrm{b}}$ becomes

$$
\begin{aligned}
\mathrm{f}_{\mathrm{b}} & =\frac{1}{2} \mathrm{~K}\left(2(\mathrm{q}(\mathrm{x}))^{2}+\left(\frac{\partial \mathrm{n}_{\mathrm{z}}}{\partial \mathrm{y}}\right)^{2}-2\left(\mathrm{n}_{\mathrm{x}} \frac{\partial \mathrm{n}_{\mathrm{z}}}{\partial \mathrm{y}} \mathrm{q}(\mathrm{x})\right)\right) \\
& =\frac{1}{2} \mathrm{~K}(\mathrm{q}(\mathrm{x}))^{2}\left(1+\mathrm{n}_{\mathrm{y}}^{2}\right),
\end{aligned}
$$

$f_{b}$ is out-of-phase with the surface energy density: when $f_{b}$ increases $\gamma$ decreases and vice versa. Using Eq. (14), we find the normal projection of the bulk Ericksen stress $\mathbf{k k}: \mathbf{T}^{E}$,

$$
\begin{aligned}
\mathbf{k k}: \mathbf{T}^{\mathrm{E}}= & -\mathrm{K}((\mathbf{n} \cdot \nabla \mathbf{n})(\mathbf{n} \cdot \mathbf{k})) \\
& \cdot((\mathbf{k} \cdot \nabla) \mathbf{n})-\mathrm{K}(\nabla \cdot \mathbf{n})((\mathbf{k} \cdot \nabla) \mathbf{n}) \cdot \mathbf{k} .
\end{aligned}
$$

Having established the director field $\mathbf{n}(\mathrm{x}, \mathrm{y})$, its associated bulk energy $f_{b}$, and the interfacial surface tension $\gamma$, we find that the normal projection of the bulk Ericksen stress, $\mathbf{k k}: \mathbf{T}^{E}$, is zero,

$$
\begin{aligned}
\mathbf{k k}: \mathbf{T}^{\mathrm{E}} & =\left(\left((\mathbf{I}-\mathbf{n n}) \cdot \frac{\partial \gamma}{\partial \mathbf{n}}\right) \cdot(\nabla \mathbf{n})^{\mathrm{T}}\right) \cdot \mathbf{k} \\
& =\mathrm{W}(\mathbf{n} \cdot \mathbf{k})\left(\left(\nabla_{\perp} \mathrm{n}_{\mathrm{y}}\right)^{\mathrm{T}}\right)=0,
\end{aligned}
$$

$\nabla_{\perp} n_{y}=0$ because $n_{y}$ depends only on $\mathrm{x}\left(\frac{\partial n_{y}}{\partial y}=0\right)$. Therefore, as the elastic correction $\mathrm{SJ}$ in the present case is only due to $\mathrm{f}_{\mathrm{b}}$,

$$
f_{b}=\left(\nabla_{s} \cdot \mathbf{T}_{\mathrm{s}}\right) \cdot \mathbf{k}
$$

\section{Liquid crystal shape equation}

To derive the capillary shape equation required to determine the surface geometry, we use the capillary pressure definition $-\mathrm{p}_{c}=-\nabla_{\mathrm{s}} \cdot \boldsymbol{\xi}=-\nabla_{\mathrm{s}} .\left(\boldsymbol{\xi}_{\perp}+\boldsymbol{\xi}_{\|}\right)$; this equation highlight the reason why the capillary vector is a very useful quantity in vectorial capillary systems. For anisotropic surfaces, the contribution from the normal component of the capillary vector 
$\xi_{\perp}$ is the classical Laplace pressure, and the contribution from the tangential component of the capillary vector, $\xi_{\|}$, is known as Herring's pressure. For anisotropic surfaces, since the capillary vector is a function of both the director field, $\mathbf{n}$, and the unit normal, $\mathbf{k}$, an additional contribution to the capillary pressure arises from director curvature due to orientation gradients. It is worth emphasizing that in this formulation the surface unit normal $\mathbf{k}$ and surface director $\mathbf{n}$ are independent but coupled, and hence their gradients are associated with different kinds of pressure. By substituting the normal $\xi_{\perp}$ and tangential $\xi_{\|}$components of the capillarity vector (Eqs. (6) and (7)), we obtain

$$
\begin{aligned}
\left(\nabla_{\mathrm{s}} \cdot \mathbf{T}_{\mathrm{s}}\right) \cdot \mathbf{k}= & \left(\gamma_{0}-\frac{\mathrm{W}}{2}(\mathbf{n} \cdot \mathbf{k})^{2}+\mathrm{W}(\mathbf{n} \cdot \mathbf{t})^{2}\right) \kappa \\
& -\mathrm{W}\left\{(\mathbf{k} \cdot \mathbf{n})\left(\mathbf{t} \cdot \frac{\mathrm{d} \mathbf{n}}{\mathrm{ds}}\right)+\mathbf{k n}:\left(\mathbf{t} \frac{\mathrm{dn}}{\mathrm{ds}}\right)\right\},
\end{aligned}
$$

where $\kappa=d \varphi / d s$ is the surface curvature, $s$ is the arc-length, and $\mathbf{t}$ is the tangential surface unit vector. Replacing Eq. (18) into Eq. (17) and using the Frank energy (Eq. (14)), the governing shape equation for the present case reads

$$
\begin{aligned}
\frac{\mathrm{K}(\mathrm{q}(\mathrm{x}))^{2}}{2 \gamma_{0}}\left(1+\mathrm{n}_{\mathrm{y}}^{2}\right)= & \left(1-\frac{\mathrm{W}}{2 \gamma_{0}}(\mathbf{n} \cdot \mathbf{k})^{2}+\frac{\mathrm{W}}{\gamma_{0}}(\mathbf{n} \cdot \mathbf{t})^{2}\right) \kappa \\
& -\frac{\mathrm{W}}{\gamma_{0}}\left\{(\mathbf{k} \cdot \mathbf{n})\left(\mathbf{t} \cdot \frac{\mathrm{dn}}{\mathrm{ds}}\right)+\mathbf{k n}:\left(\mathbf{t} \frac{\mathrm{dn}}{\mathrm{ds}}\right)\right\},
\end{aligned}
$$

where $\mathrm{K} / \gamma_{0}$ represents the elasto-capillary length scale which is the ratio of bulk elasticity to isotropic surface tension, and $\mathrm{W} / \gamma_{0}$ represents the dimensionless or scaled anchoring coefficient. This equation shows that the surface shape is the result of the balance between bulk Frank elasticity, surface tension, and surface anchoring effects. The anchoring term can drive the surface wrinkling in cholesteric liquid crystals since for a fixed director field, the total surface energy can only be minimized by distorting the interface. The surface shape also depends on the relative influence of the elastic contributions to the capillary pressure. The relative influence of elastic contributions compared to anchoring can be evaluated by comparing the extrapolation length, $\ell_{e x}=\frac{K}{W}$ and the helix pitch, P. For biological cholesteric liquid crystals with significant anchoring that unravels the helix and with a large enough pitch (order of micrometers), the elastic contribution to the shape equation is not significant as the extrapolation length is much less than the helix pitch: $\ell_{e x}<P$. By increasing the helix pitch through hydration, the bulk elastic contribution $\left(\frac{K\left(1+n_{\mathrm{y}}^{2}\right)}{2 \gamma_{0}}\right)$ decreases and eventually essentially vanishes. Nevertheless in order to exactly determine the surface profile, it is thus essential to consider the bulk elastic contribution together with the three surface capillary pressures in the shape equation (Eq. (19)).

\section{Chiral surface shape equation and material parameters}

Considering the specified splay-bend director $\mathbf{n}(\mathrm{x})$ $=(\cos \mathrm{qx}, \sin \mathrm{qx}, 0)$, surface unit normal $\mathbf{k}(\mathrm{x})=(\cos \varphi(\mathrm{x})$, $\sin \varphi(\mathrm{x}), 0)$, unit tangent $\mathbf{t}(\mathrm{x})=(\sin \varphi(\mathrm{x}),-\cos \varphi(\mathrm{x}), 0)$, and using the definitions,

$$
\frac{\mathrm{dx}}{\mathrm{ds}}=\sin \varphi, \frac{\mathrm{d} \mathbf{n}}{\mathrm{ds}}=\frac{\mathrm{d} \mathbf{n}}{\mathrm{dx}} \frac{\mathrm{dx}}{\mathrm{ds}}=\frac{\mathrm{dn}}{\mathrm{dx}} \sin \varphi, \kappa=\frac{\mathrm{d} \varphi}{\mathrm{dx}} \sin \varphi
$$

give the governing shape equation

$$
\frac{\mathrm{d} \varphi}{\mathrm{dx}}=\frac{\frac{\mathrm{Kq}^{2}(\mathrm{x})}{2 \gamma_{0}}\left(1+\mathrm{n}_{\mathrm{y}}^{2}\right)+\frac{\mathrm{q}(\mathrm{x}) \mathrm{w}}{\gamma_{0}} \sin (\varphi)\left\{\sin ^{2}(\varphi-\mathrm{q}(\mathrm{x}) \mathrm{x})-\cos ^{2}(\varphi-\mathrm{q}(\mathrm{x}) \mathrm{x})\right\}}{\sin (\varphi)\left\{1-\frac{\mathrm{w}}{2 \gamma_{0}} \cos ^{2}(\varphi-\mathrm{q}(\mathrm{x}) \mathrm{x})+\frac{\mathrm{w}}{\gamma_{0}} \sin ^{2}(\varphi-\mathrm{q}(\mathrm{x}) \mathrm{x})\right\}}
$$

The shape equation is a nonlinear first order ordinary differential equation (ODE) for the normal angle $\varphi\left(x, K / \gamma_{0}, W / \gamma_{0}, q(x)\right)$, where the surface length scale $\mathrm{L}$, the elasto-capillary length $\mathrm{K} / \gamma_{0}$, extrapolation length $\mathrm{W} / \gamma_{0}$, wave vector $\mathrm{q}$, and pitch $\mathrm{P}$ are

$$
0<\mathrm{x}<\mathrm{L}=4.8 \mu \mathrm{m}, 1 \mathrm{~nm}<\frac{\mathrm{K}}{\gamma_{0}}<100 \mathrm{~nm},-2<\frac{\mathrm{W}}{\gamma_{0}}<0, \mathrm{q}(\mathrm{x})=\frac{2 \pi}{\mathrm{P}(\mathrm{x})}, \mathrm{P}(\mathrm{x})=\mathrm{P}_{\text {dry }}+\left(\mathrm{P}_{\text {wet }}-\mathrm{P}_{\text {dry }}\right) \frac{\mathrm{x}}{\mathrm{L}} .
$$

The nonlinear ODE with periodic coefficients is solved using the AUTO software. ${ }^{57}$ The surface relief is then obtained from

$$
\mathrm{h}(\mathrm{x})=\int_{0}^{\mathrm{x}} \cot \varphi \mathrm{dx^{ \prime }} .
$$

The boundary condition at $\mathrm{x}=0$ is $\left.\varphi\right|_{\mathrm{x}=0}=\frac{\pi}{2}$, consistent with the adopted sign of $\mathrm{W} / \gamma_{0}$. The generic features of the amplitude profile $\mathrm{h}(\mathrm{x})$ and its periodicity $\mathrm{h}(\mathrm{x})=\mathrm{h}(\mathrm{x}+\lambda)$ are the important outputs of the model. The four significant parameters influencing $\mathrm{h}(\mathrm{x})$ are the following: (i) The scaled anchoring coefficient: $\mathrm{W} / \gamma_{0}$.

(ii) The elasto-capillary length scale: $\mathrm{K} / \gamma_{0}$.

(iii) The micron scale pitch in dry state: $\mathrm{P}_{\text {dry }}$.

(iv) The hydration-driven pitch gradient: $\left(\mathrm{P}_{\mathrm{wet}}-\mathrm{P}_{\mathrm{dry}}\right) / \mathrm{L}$.

For the nematic-isotropic interface, the scaled anchoring coefficient $\mathrm{W} / \gamma_{0}$ is of the order of $0.01 .^{58}$ The anchoring strength $\mathrm{W}$ at the nematic-air interface is about several orders of magnitude larger than the anchoring strength at the nematicisotropic interface. However, as the surface tension at the nematic-air interface is higher than the surface tension at the 
nematic-isotropic interface, ${ }^{6,29}$ the scaled anchoring coefficient $\mathrm{W} / \gamma_{0}$ is taken to be in the range $-0.1<\mathrm{W} / \gamma_{0}<-0.01$. For typical cholesteric liquid crystals, the elasto-capillary length scale $\mathrm{K} / \gamma_{0}$ is usually in the order of few nanometers (an order of magnitude estimation of the elastic constant $\mathrm{K}$, and the surface tension $\gamma_{0}$ gives $\mathrm{K} \approx 10^{-11} \mathrm{~J} / \mathrm{m}$ and $\gamma_{0} \approx 10^{-2} \mathrm{~J} / \mathrm{m}^{2}$ ). So, the elasto-capillary length scale, $\mathrm{K} / \gamma_{0}$, is taken to be in the range $1 \mathrm{~nm}<\mathrm{K} / \gamma_{0}<100 \mathrm{~nm}$. The helix deformation through tangential water gradient is described by a linear pitch profile so that the pitch increases from $\mathrm{P}_{\text {dry }}$ to $\mathrm{P}_{\text {wet }}$ along the wave propagating direction. $\mathrm{P}_{\mathrm{dry}}$ and $\left(\mathrm{P}_{\text {wet }}-\mathrm{P}_{\mathrm{dry}}\right) / \mathrm{L}$ are taken to be in the range $0.5 \mu \mathrm{m}<\mathrm{P}_{\text {dry }}<3 \mu \mathrm{m}$ and $0.1<\left(\mathrm{P}_{\mathrm{wet}}-\mathrm{P}_{\mathrm{dry}}\right) / \mathrm{L}<0.3$, respectively.

\section{RESULTS AND DISCUSSION}

\section{A. Free surface profile}

Fig. 3 shows the amplitude $h_{\max }$ profile for increasingly scaled values of the anchoring $\mathrm{W} / \gamma_{0}$. The amplitude increases with increasing $\mathrm{W} / \gamma_{0}$. For constant pitch, the periodicity of the undulation is constant and equal to the helix pitch $\mathrm{P}^{31} \mathrm{In}$ the case of a cholesteric with a linearly varying pitch $\mathrm{P}(\mathrm{x})$, the periodicity increases quadratically along $\mathrm{x}$, due to the integral relation between surface relief $h(x)$ and the cotangent of the normal angle $\varphi$. The $h$ periodicity is independent of $\mathrm{W} / \gamma_{0}$. Fig. 4 shows the corresponding surface profiles $\mathrm{h}(\mathrm{x})$ as the elasto-capillary length scale $\mathrm{K} / \gamma_{0}$ increases. The surface amplitude and periodicity remain essentially constant. One can infer that for particular values of the anchoring coefficient and the helix pitch, the elastic contribution to the surface shape is not significant and can be neglected. Fig. 5 illustrates the variation of the amplitude and periodicity with the initial helix pitch $\mathrm{P}_{\text {dry }}$. The figure shows that higher $\mathrm{P}_{\text {dry }}$ increases the periodicity. Since this pitch sets the scale of the pattern, increasing the base pitch increases the amplitude, which is consistent with the constant pitch case (see Refs. 32 and 31). The reason behind this fact is that the Laplace pressure scales with $\mathrm{h} \gamma_{0} / \mathrm{P}^{2}$, while the balancing director pressure scales with W/P. Fig. 6 shows the corresponding surface profiles $\mathrm{h}(\mathrm{x})$ for increasing $\left(\mathrm{P}_{\mathrm{wet}}-\mathrm{P}_{\text {dry }}\right) /$ L. Both amplitude and periodicity increase but the effect increases with " $x$ " since it is the gradient factor of the pitch.

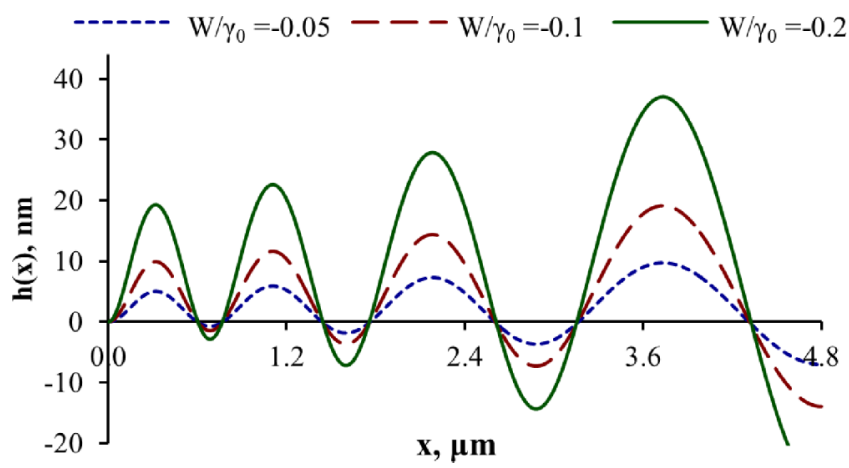

FIG. 3. Surface profile $\mathrm{h}(\mathrm{x})$ for $\mathrm{K} / \gamma_{0}=1 \mathrm{~nm}, \mathrm{P}_{\mathrm{dry}}=1.2 \mu \mathrm{m}$, and $\left(\mathrm{P}_{\text {wet }}\right.$ $\left.-\mathrm{P}_{\text {dry }}\right) / \mathrm{L}=0.2$ and different values of $\mathrm{W} / \gamma_{0}=-0.05,-0.1$, and -0.2 , showing the increase of the resultant surface amplitude $h(x)$ through increase in the anchoring strength $\mathrm{W} / \gamma_{0}$.

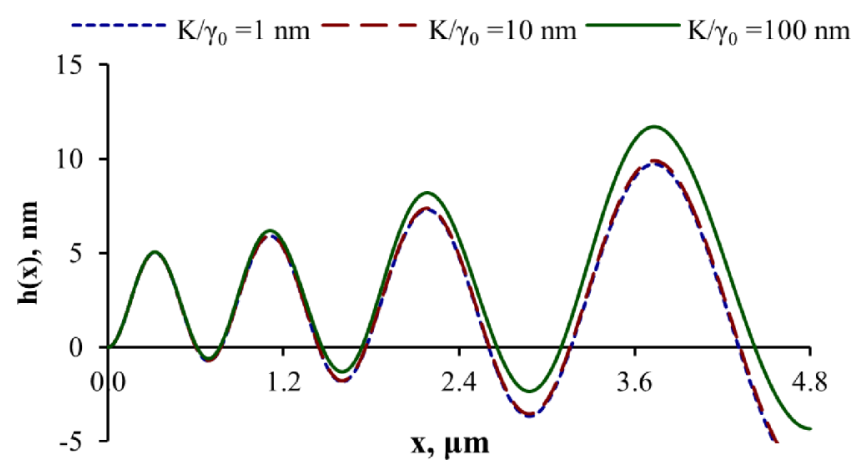

FIG. 4. Surface profile $\mathrm{h}(\mathrm{x})$ for $\mathrm{W} / \gamma_{0}=-0.05, \mathrm{P}_{\mathrm{dry}}=1.2 \mu \mathrm{m}$, and $\left(\mathrm{P}_{\text {wet }}\right.$ $\left.-\mathrm{P}_{\text {dry }}\right) / \mathrm{L}=0.2$ and different values of $\mathrm{K} / \gamma_{0}=1 \mathrm{~nm}, 10 \mathrm{~nm}$, and $100 \mathrm{~nm}$, showing the small shift of the resultant surface amplitude $h(x)$ through increase in the elasto-capillary length scale $\mathrm{K} / \gamma_{0}$.

\section{B. Pressure-curvature relations}

As mentioned above, the main contributions to the capillary pressure arise from area changes (Laplace pressure), area rotation (Herrings pressure), director curvature, and from the bulk stress jump (known as the elastic correction). ${ }^{33}$ The Laplace capillary pressure, $\mathrm{P}_{\text {dilation, }}$, is the resistant term (increasing energy with increasing area), while the Herring's capillary pressure, $\mathrm{P}_{\text {rotation}}$, and the director curvature pressure, $\mathrm{P}_{\text {director }}$, are the driving forces that undulate the interface.

Rearranging Eq. (20b) gives the three surface pressures and the bulk stress jump as a function of " $x$,"

$$
\begin{aligned}
& \underbrace{\sin (\varphi) \frac{\mathrm{d} \varphi}{\mathrm{dx}}}_{P_{\text {dilation }}}-\underbrace{\left\{\frac{\mathrm{W}}{2 \gamma_{0}} \cos ^{2}(\varphi-\mathrm{q}(\mathrm{x}) \mathrm{x})+\frac{\mathrm{W}}{\gamma_{0}} \sin ^{2}(\varphi-\mathrm{q}(\mathrm{x}) \mathrm{x})\right\} \frac{\mathrm{d} \varphi}{\mathrm{dx}}}_{P_{\text {rotation }}} \\
& -\underbrace{\frac{\mathrm{q}(\mathrm{x}) \mathrm{W}}{\gamma_{0}} \sin (\varphi)\left\{\sin ^{2}(\varphi-\mathrm{q}(\mathrm{x}) \mathrm{x})-\cos ^{2}(\varphi-\mathrm{q}(\mathrm{x}) \mathrm{x})\right\}}_{\mathrm{P}_{\text {director }}} \\
& =\underbrace{\frac{\mathrm{Kq}^{2}(\mathrm{x})}{2 \gamma_{0}}\left(1+\mathrm{n}_{\mathrm{y}}^{2}\right)}_{\text {Stress Jump. SJ }},
\end{aligned}
$$

where we have introduced the scaled pressures (divided by isotropic tension $\gamma_{0}$ ). The director curvature term reflects the

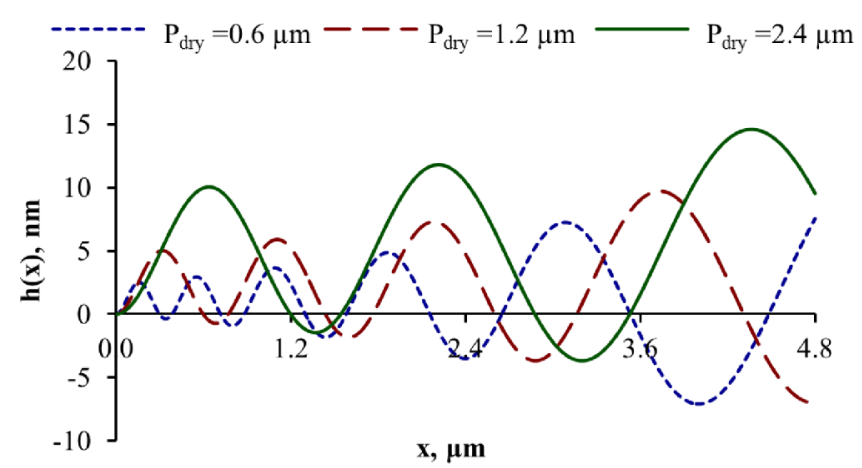

FIG. 5. Surface profile $\mathrm{h}(\mathrm{x})$ for $\mathrm{W} / \gamma_{0}=-0.05, \mathrm{k} / \gamma_{0}=1 \mathrm{~nm}$, and $\left(\mathrm{P}_{\text {wet }}\right.$ $\left.-\mathrm{P}_{\mathrm{dry}}\right) / \mathrm{L}=0.2$ and different values of $\mathrm{P}_{\mathrm{dry}}=0.6 \mu \mathrm{m}, 1.2 \mu \mathrm{m}$, and $2.4 \mu \mathrm{m}$, showing the increase of the resultant surface amplitude $h(x)$ through increase in the pitch at dry state $\mathrm{P}_{\text {dry }}$. 


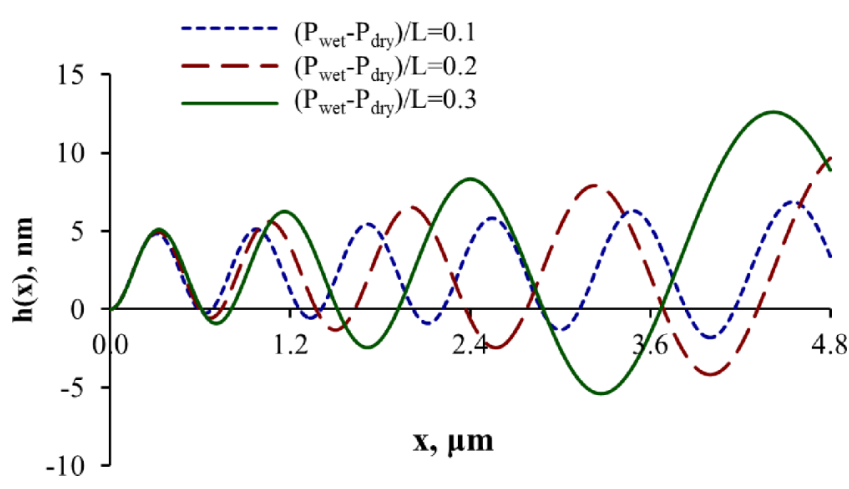

FIG. 6. Surface profile $\mathrm{h}(\mathrm{x})$ for $\mathrm{W} / \gamma_{0}=-0.05, \mathrm{k} / \gamma_{0}=1 \mathrm{~nm}$, and $\mathrm{P}_{\text {dry }}$ $=1.2 \mu \mathrm{m}$ and different values of $\left(\mathrm{P}_{\mathrm{wet}}-\mathrm{P}_{\text {dry }}\right) / \mathrm{L}=0.1,0.2$, and 0.3 , showing the increase of the resultant surface amplitude $h(x)$ through increase in the pitch gradient rate $\left(\mathrm{P}_{\mathrm{wet}}-\mathrm{P}_{\mathrm{dry}}\right) / \mathrm{L}$.

unique anisotropic nature of chiral liquid crystals through the orientation contribution to the surface energy. The director variation in the bulk that contains orientation gradient contributions is the origin of the stress jump term.

Fig. 7 identifies and illustrates the mechanisms behind chiral wrinkling using the three pressures and the bulk stress jump. The scaled surface pressure contributions and the stress jump are plotted as a function of " $x$ " for representative values of $\mathrm{K} / \gamma_{0}, \mathrm{~W} / \gamma_{0}, \mathrm{P}_{\text {dry }}$, and $\left(\mathrm{P}_{\mathrm{wet}}-\mathrm{P}_{\text {dry }}\right) / \mathrm{L}$. For an increasing helix pitch, the amplitudes of all contributions decrease along $\mathrm{x}$, because the director spatial periodicity increases. The Herring's capillary pressure, $P_{\text {rotation, }}$ and the bulk SJ are an order of magnitude smaller than the Laplace capillary pressure, $\mathrm{P}_{\text {dilation }}$, and the director curvature, $\mathrm{P}_{\text {director }}$. The figure demonstrates that the Herring's pressure $\mathrm{P}_{\text {rotation }}$ is always positive, the stress jump is always negative, and they change their phase along each cycle, such that in the first circle, rotation and director curvature are in-phase while dilation and stress jump are out-of-phase, and in the second circle, rotation and dilation are in-phase while director and stress jump are out-of-phase. The SJ decays along $\mathrm{x}$, and its contribution becomes insignificant when the helix pitch increases driven by the tangential water gradient. The key observation from these pressure profiles is that dilation and director curvature pressures are always out-of-phase, and since dilation (increase area) resists wrinkling, the director curvature pressure is the main driving force behind nano-wrinkling.

Polar plots of pressure as a function of director angle reveal the intimate connection between forces and director orientation. The polar plots of the three scaled capillary pressures and stress jump as a function of the anchoring coefficient $\mathrm{W} / \gamma_{0}$, the elasto-capillary length scale $\mathrm{K} / \gamma_{0}$, and the pitch gradient profile $\left(\mathrm{P}_{\text {dry }}\right.$ and $\left.\left(\mathrm{P}_{\text {wet }}-\mathrm{P}_{\text {dry }}\right) / \mathrm{L}\right)$ are shown in Figs. 8-11, respectively. The angular coordinate is the director field $\theta$. Fig. 8 shows that all surface capillary pressures grow as the anchoring increases, while the stress jump remains constant. Changing $\mathrm{W} / \gamma_{0}$ does not affect the degree of asymmetry between the lobes of the three pressures. Fig. 9 shows that as the elasto-capillary length scale $\mathrm{K} / \gamma_{0}$ increases, the Laplace pressure $\mathrm{P}_{\text {dilation }}$, Herring's pressure $\mathrm{P}_{\text {rotation }}$, and stress jump increase, whereas the director pressure $\mathrm{P}_{\text {director }}$ remains constant. This is due to the fact that the director pressure depends on the anchoring strength and chirality and is independent of the elasto-capillary length scale. Varying $\mathrm{W} / \gamma_{0}$ changes the degree of asymmetry between the lobes of the Laplace pressure $\mathrm{P}_{\text {dilation }}$ and Herring's pressure $\mathrm{P}_{\text {rotation. }}$. Figs. 10 and 11 illustrate that a decrease in chirality (increasing $\mathrm{P}(\mathrm{x})$ ) through a raise of either $\mathrm{P}_{\text {dry }}$ or $\left(\mathrm{P}_{\mathrm{wet}}-\mathrm{P}_{\text {dry }}\right) / \mathrm{L}$ reduces all capillarity pressures and the stress jump as the wavelength of the undulation increases. Changing $\mathrm{P}(\mathrm{x})$ does not affect the degree of asymmetry between the lobes of the pressures and stress jump.

\section{Nano-wrinkling scaling law}

For free surface relief of cholesteric liquid crystal with constant pitch, we previously presented a theoretical scaling law expressing for the maximum undulation amplitude $\mathrm{h}_{\max }$ as a function of anchoring strength and chirality, ${ }^{31}$

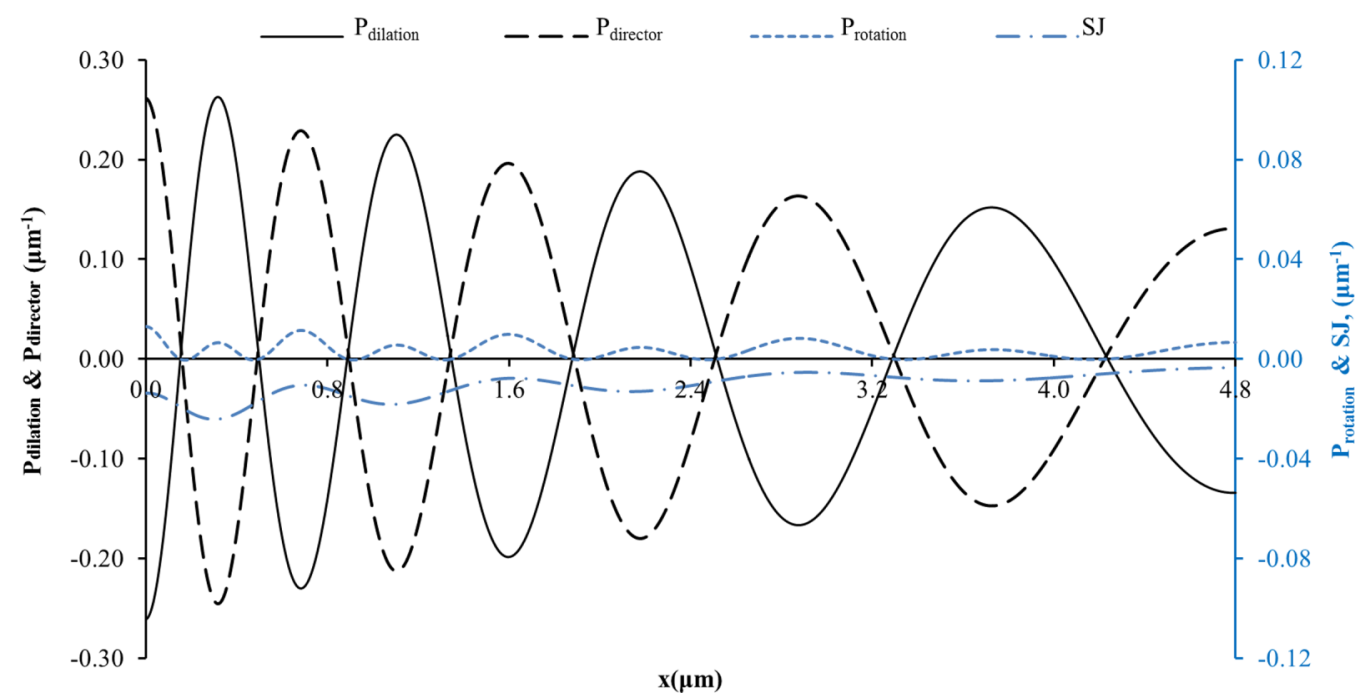

FIG. 7. Pressure profiles for $\mathrm{P}_{\text {dilation }}, \mathrm{P}_{\text {rotation }}, \mathrm{P}_{\text {director }}$ and SJ as a function of distance " $\mathrm{x}$ " for $\mathrm{W} / \gamma_{0}=-0.05, \mathrm{k} / \gamma_{0}=1 \mathrm{~nm}, \mathrm{P}_{\mathrm{dry}}=1.2 \mu \mathrm{m}$, and $\left(\mathrm{P}_{\mathrm{wet}}-\mathrm{P}_{\mathrm{dry}}\right) / \mathrm{L}=0.2$. The figure displays that pressure extrema occur at planar and homeotropic orientation. When the director angle is $\pi / 4<\theta<\pi / 2$, dilation and rotation are in-phase, and when $0<\theta<\pi / 4$, rotation and director curvature are in-phase. 


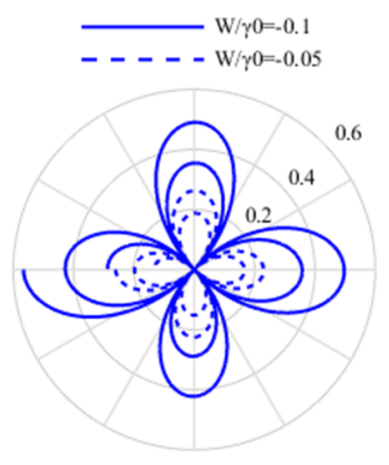

(a)

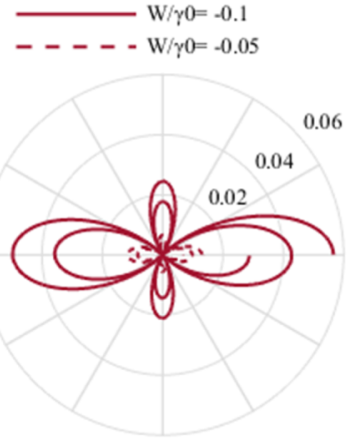

(b)

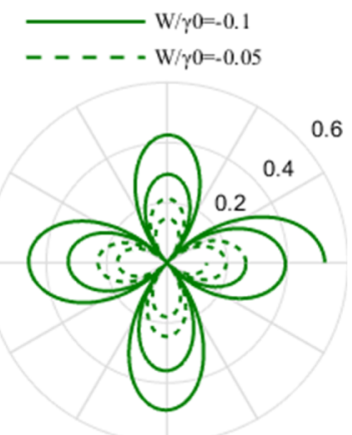

(c)

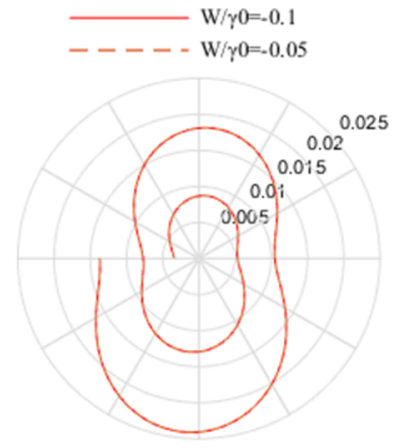

(d)

FIG. 8. Polar plots of the three scaled capillary pressures and stress jump: (a) $\mathrm{P}_{\text {dilation }}\left(\mu \mathrm{m}^{-1}\right)$, (b) $\mathrm{P}_{\text {rotation }}\left(\mu \mathrm{m}^{-1}\right)$, (c) $\mathrm{P}_{\text {director }}\left(\mu \mathrm{m}^{-1}\right)$, and (d) SJ for two different values of $\mathrm{W} / \gamma_{0}=-0.05,-0.1$ where $\mathrm{K} / \gamma_{0}=1 \mathrm{~nm}, \mathrm{P}_{\mathrm{dry}}=1.2 \mu \mathrm{m}$, and $\left(\mathrm{P}_{\mathrm{wet}}-\mathrm{P}_{\mathrm{dry}}\right) / \mathrm{L}=0.2$. The angular coordinate is the director field $\theta$.

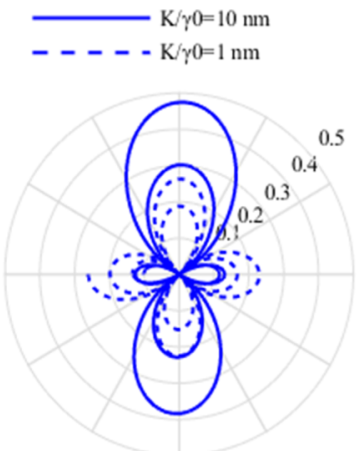

(a)

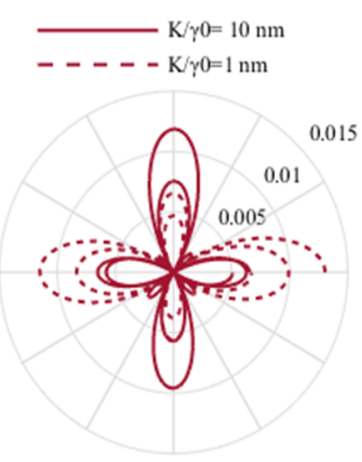

(b)

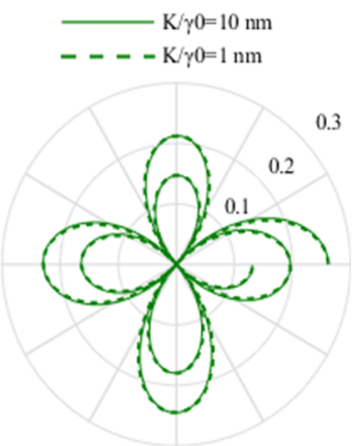

(c)

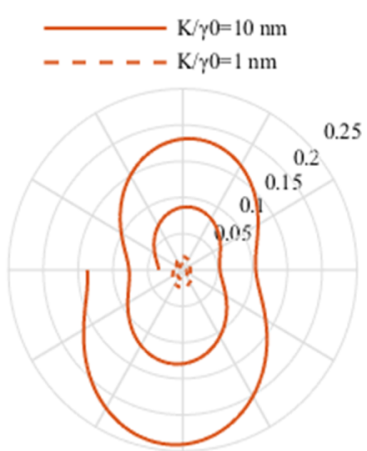

(d)

FIG. 9. Polar plots of the three scaled capillary pressures and stress jump: (a) $\mathrm{P}_{\text {dilation }}\left(\mu \mathrm{m}^{-1}\right)$, (b) $\mathrm{P}_{\text {rotation }}\left(\mu \mathrm{m}^{-1}\right)$, (c) $\mathrm{P}_{\text {director }}\left(\mu \mathrm{m}^{-1}\right)$, and (d) $\mathrm{SJ}$ for two different values of $\mathrm{K} / \gamma_{0}=1,10 \mathrm{~nm}$ where $\mathrm{W} / \gamma_{0}=-0.05, \mathrm{P}_{\text {dry }}=1.2 \mu \mathrm{m}$, and $\left(\mathrm{P}_{\mathrm{wet}}-\mathrm{P}_{\mathrm{dry}}\right) / \mathrm{L}=0.2$. The angular coordinate is the director field $\theta$.

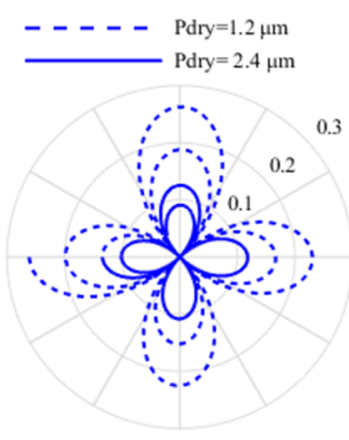

(a)

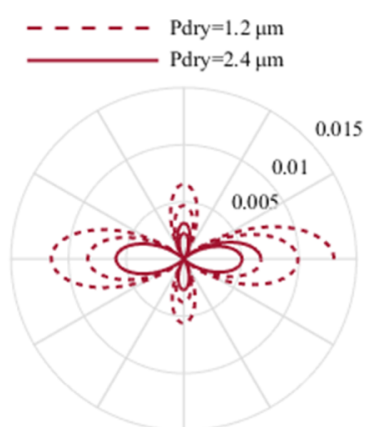

(b)

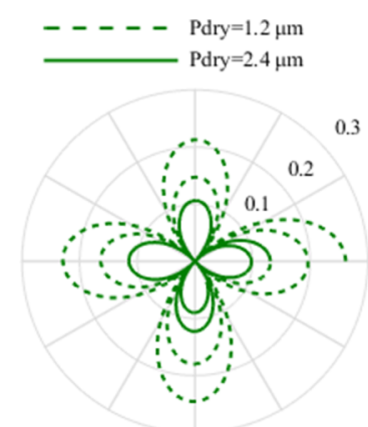

(c)

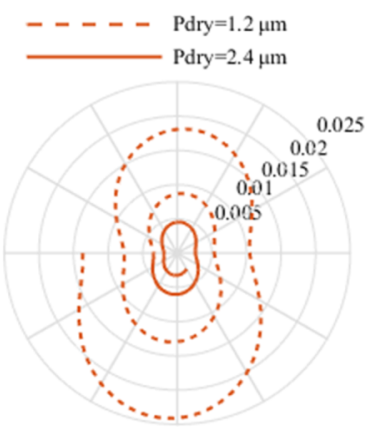

(d)

FIG. 10. Polar plots of the three scaled capillary pressures and stress jump: (a) $P_{\text {dilation }}\left(\mu \mathrm{m}^{-1}\right)$, (b) $\mathrm{P}_{\text {rotation }}\left(\mu \mathrm{m}^{-1}\right)$, (c) $\mathrm{P}_{\text {director }}\left(\mu \mathrm{m}^{-1}\right)$, and (d) SJ for two different values of $\mathrm{P}_{\mathrm{dry}}=1.2,2.4 \mu \mathrm{m}$ where $\mathrm{K} / \gamma_{0}=1 \mathrm{~nm}, \mathrm{~W} / \gamma_{0}=-0.05$, and $\left(\mathrm{P}_{\mathrm{wet}}-\mathrm{P}_{\mathrm{dry}}\right) / \mathrm{L}=0.2$. The angular coordinate is the director field $\theta$.

$$
\mathrm{h}_{\max }=\frac{\frac{\mathrm{WP}}{\gamma_{0}}}{1+\frac{\mathrm{WP}}{\delta}} .
$$

The numerical results indicated that $\delta=10.71\left(\frac{W}{\gamma_{0}}\right)^{-1.02}$. This prediction shows that the undulation amplitude is essentially a linear function of the scaled anchoring $\mathrm{W} / \gamma_{0}$ and the helical pitch: $h_{\max }=0.085 P_{0}\left(\frac{W}{\gamma_{0}}\right)$, which is consistent with the nano-scale surface structures that have been experimentally observed in a variety of polymeric and biological CLC. Recently, Yoshioka et al. $^{59}$ showed that the amplitude and the period of interface distortions of cholesteric droplets are determined by the helical pitch and independent of the droplet size. They stated that the amplitude monotonically increases with the pitch length; we note that Bernardino et al. claimed that the cholesteric-isotropic interface undulations scale with square root of the pitch. ${ }^{60}$ For the periodic surface relief found in cholesteric liquid crystal with water-induced varying pitch, we also find a revealing close form expression for the $\mathrm{x}$-dependent profile amplitude $\mathrm{A}$ ( $\mathrm{A}$ is the amplitude between the upper and the lower envelope of the periodic surface undulations) as a function of the scaled anchoring $\mathrm{W} / \gamma_{0}$, the helix pitch at dry state $\mathrm{P}_{\mathrm{dry}}$, and the pitch gradient $\left(\mathrm{P}_{\text {wet }}-\mathrm{P}_{\mathrm{dry}}\right) / \mathrm{L}$, 


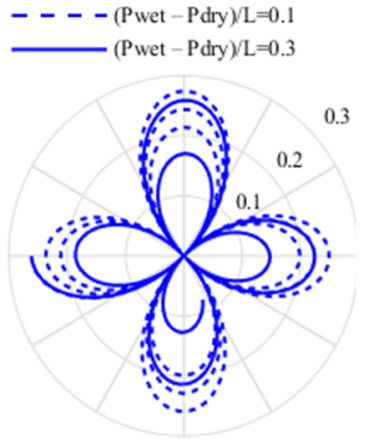

(a)

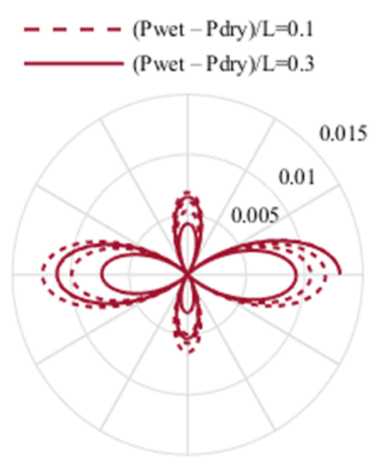

(b)

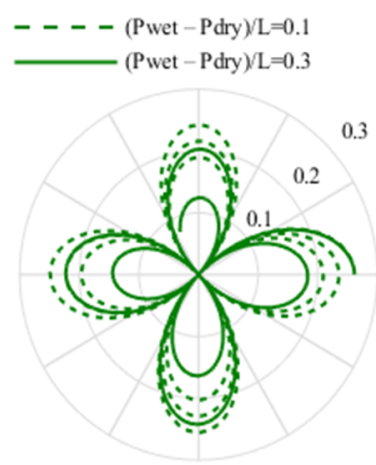

(c)

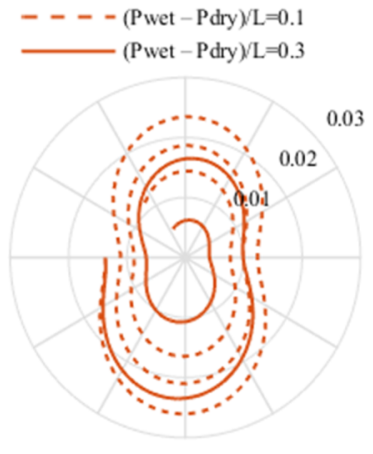

(d)

FIG. 11. Polar plots of the three scaled capillary pressures and stress jump: (a) $\mathrm{P}_{\text {dilation }}\left(\mu \mathrm{m}^{-1}\right)$, (b) $\mathrm{P}_{\text {rotation }}\left(\mu \mathrm{m}^{-1}\right)$, (c) $\mathrm{P}_{\text {director }}\left(\mu \mathrm{m}^{-1}\right)$, and (d) SJ for two different values of $\left(\mathrm{P}_{\mathrm{wet}}-\mathrm{P}_{\mathrm{dry}}\right) / \mathrm{L}=0.1$ and 0.3 where $\mathrm{K} / \gamma_{0}=1 \mathrm{~nm}, \mathrm{~W} / \gamma_{0}=-0.05$, and $\mathrm{P}_{\text {dry }}=1.2 \mu \mathrm{m}$. The angular coordinate is the director field $\theta$.

$$
\mathrm{A}=\alpha_{1}\left(\frac{\mathrm{W}}{\gamma_{0}}\right) \mathrm{P}_{\text {dry }}+\alpha_{2}\left(\frac{\mathrm{W}}{\gamma_{0}}\right)\left(\frac{\mathrm{P}_{\text {wet }}-\mathrm{P}_{\text {dry }}}{\mathrm{L}}\right) \mathrm{x},
$$

where $\alpha_{1}=-0.07$ and $\alpha_{2}=0.13$, found from numerical simulations. In accordance with the scaling law presented for cholesteric liquid crystal with constant pitch (Eq. (24)), the estimate is that the amplitude is essentially a linear function of the scaled anchoring and the variable helix pitch. As shown in Fig. 12, this result is consistent with experimental data from optical and scanning force microscopy of free surface of a chiral liquid crystal, for which the ratio of period and depth is approximately constant. ${ }^{61}$

\section{Diffraction grating}

When the periodicity of the surface undulations is of the same order of the incident light wavelength, the structure has the potential to generate iridescence and colors through the diffraction grating mechanism. Structural color in floral plants originates mainly from ordered surface diffraction gratings that scatter incident light in the plane perpendicular to the direction of the periodic undulations according to the grating equation ${ }^{14}$

$$
\mathrm{m} \lambda=\frac{\mathrm{P}_{0}}{2}\left(\sin \theta_{\mathrm{i}}-\sin \theta_{\mathrm{d}}\right),
$$

where $\theta_{\mathrm{i}}$ and $\theta_{\mathrm{d}}$ are the angles of incidence and diffraction which govern the angular locations of the principal intensity

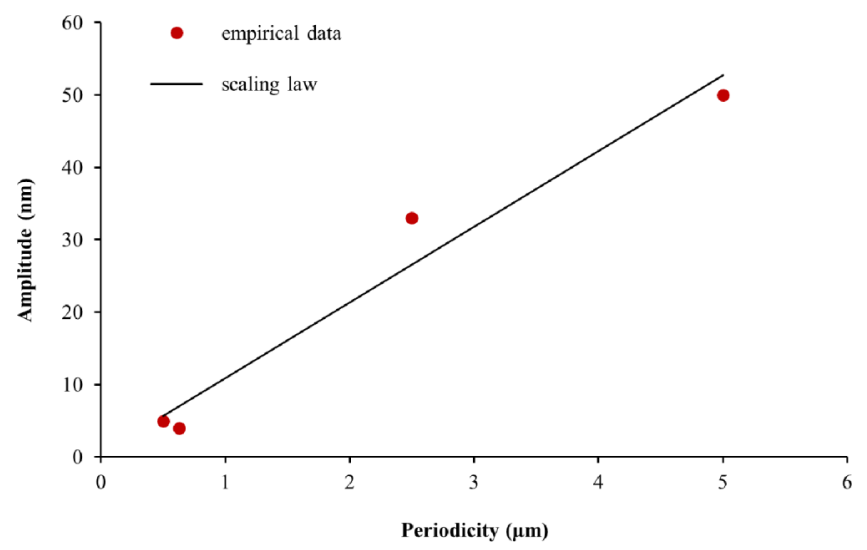

FIG. 12. Validation of the scaling law with experimentally observed nanoscale undulations at CLCs free surface. ${ }^{61}$ maxima for the diffracted incident light with wavelength $\lambda$, and $m$ is the diffraction order. When the incident light is along the helix axis, mainly first order reflection occurs. While for oblique incidence of light or the distorted helical structures, higher order reflections can happen. ${ }^{62}$ According to Eq. (26), for any given value of the angle of incidence $\theta_{i}$, each wavelength $\lambda$ scatters into different angular directions. When the incident light is white light, different colors disperse in the perpendicular direction of the periodic structure, making the surface iridescent. Changing the angle of incidence, $\theta_{i}$, can yield a variation in the peak wavelength. It has been shown that increasing the angle of incidence in a jeweled beetle, Chrysina gloriosa causes a red shift in wavelength from $525 \mathrm{~nm}$ at normal incidence to $556 \mathrm{~nm}$ in oblique illumination. ${ }^{3}$ Hence, Eq. (26) provides an important new relation between functionality and structure of nano-wrinkled chiral surfaces of plywoods. In this paper, the interaction of light with the surface nanostructure is computationally investigated with FDTD simulation using the OptiFDTD12 software $^{63}$ that solves the differential form of Maxwell's equations by discretizing time and space on a finite rectangular grid. Diffraction from periodic nanostructures is simulated in two dimensions, while considering "perfectly matched layer," PML, on top and bottom and "periodic boundary conditions," PBCs, on the sides of the computational domain. The near-zone scattered field on the top surface of the simulation domain determines the reflection intensity. We considered the refractive index $n$ of the nanostructure scales to be 1.55 . To determine the reflection and transmission intensity, observation lines are positioned on the top surface and the bottom of the diffracting interface (see Figs. 13(a) and 13(b)). For the surface structure with constant pitch, we assumed that the grating pitch is $1.2 \mu \mathrm{m}$, the grating depth is $20 \mathrm{~nm}$, and the film thickness is $200 \mathrm{~nm}$. For the surface structure with varying pitch, we assumed the grating pitch to grow from $\mathrm{P}_{\text {dry }}=1.2 \mu \mathrm{m}$ to $\mathrm{P}_{\text {wet }}=2.2 \mu \mathrm{m}$ along the $\mathrm{x}$-direction; the grating depth and the film thickness are $20 \mathrm{~nm}$ and $200 \mathrm{~nm}$, respectively. As shown in Figs. 13(d) and 13(e), the scattering patterns in the surface structure with constant pitch and with varying pitch are different. It should be noted that the electric field intensity in the surface structure with varying pitch, as compared with that in the flat surface structure (Fig. 13(c)) and in the structure with constant pitch (Fig. 13(d)), becomes weaker. 


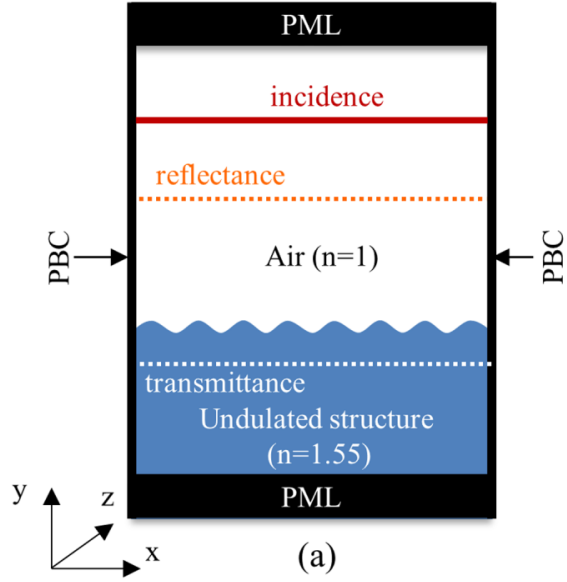

(a)

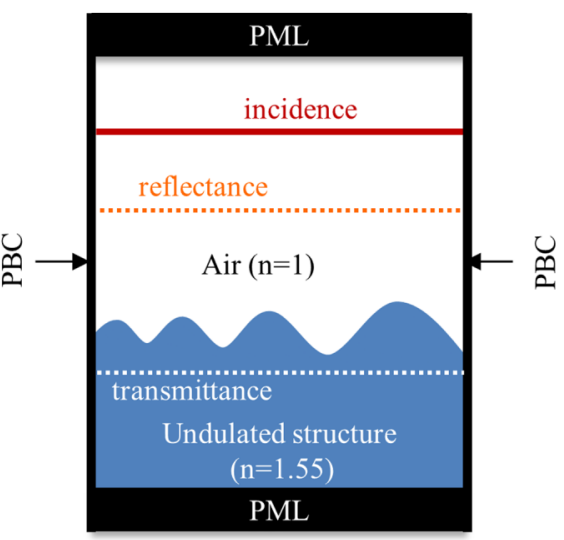

(b)

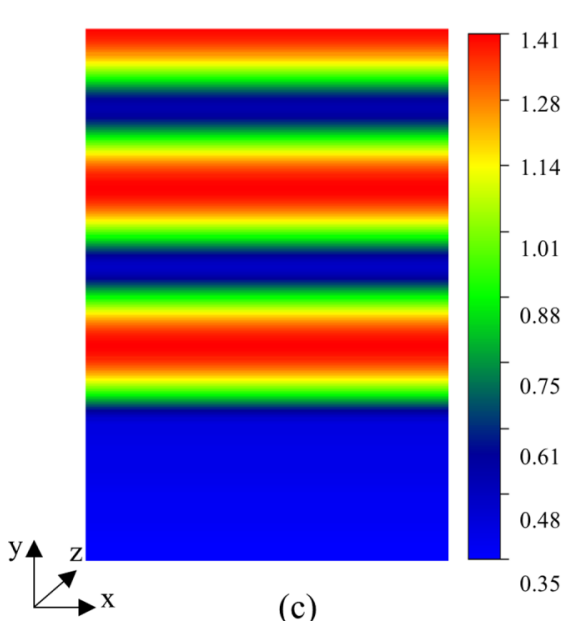

(c)

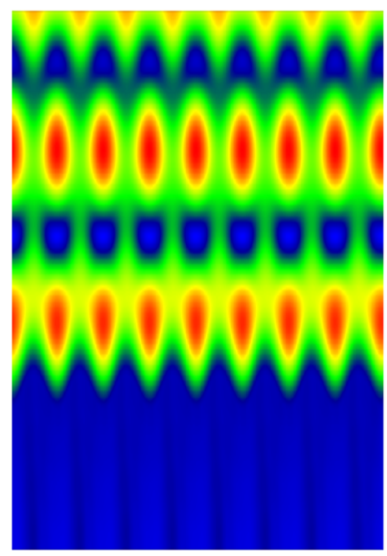

(d)

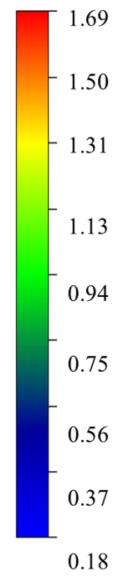

0.18

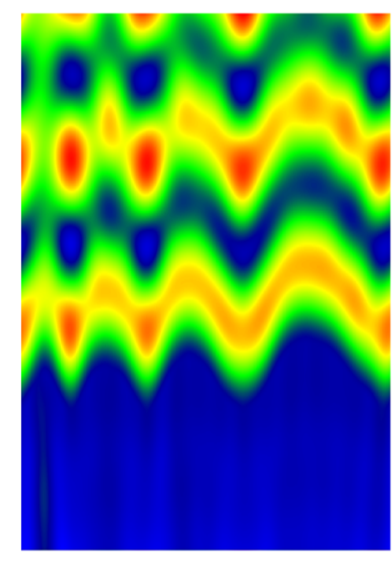

(e)

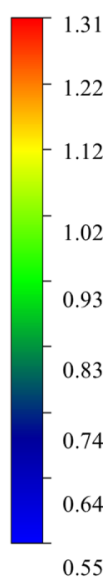

0.55

FIG. 13. Optical FDTD simulations of the surface nanostructures. (a) 2D schematic of the computational domain for air-CCLC interface with constant pitch (b) 2D schematic of the computational domain for air-CCLC interface with water-induced varying pitch. Perfectly matched layers are considered at the top and bottom of the grating structure; periodic boundary conditions are used on the left and right sides of the structure. Scattered electric field of $480 \mathrm{~nm}$ normal incident light in the $x$-y plane for (c) CCLC-air flat surface, (d) CCLC-air surface nanostructure with constant pitch, and (e) CCLC-air surface nanostructure with variable pitch, illustrating differences in the scattering behavior.

This shows that the helix pitch growth through hydration could decrease light scattering, as the reflection energy is distributed over a wide wavelength range. Fig. 14 shows the simulated optical reflectance for the nanostructures with constant and varying pitch as a function of wavelength $\lambda$ for different observation angles $\theta_{\mathrm{D}}$. The structure with constant helix pitch reflects a band of 440-525 $\mathrm{nm}$ of the incoming white light (see Fig. 14(a)). The reflectivity peak red shifts
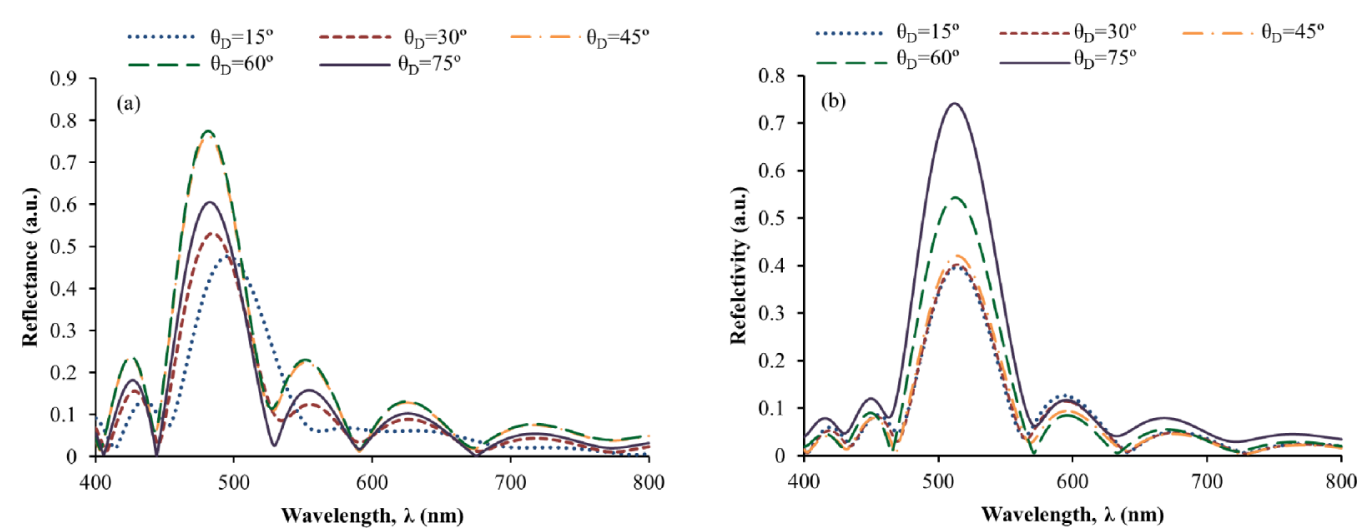

FIG. 14. FDTD simulation results for the reflectivity of the two surface nanostructures with constant pitch and varying pitch as a function of the wavelength $\lambda$. The reflectance is numerically calculated for two different structures by measuring the scattered field power normalized by the incident power. Reflectivity as a function of observation angles at $480 \mathrm{~nm}$ for the nanostructures (a) with constant and (b) varying pitch. For CCLC with constant pitch, the reflectivity peak at $\theta_{\mathrm{D}}=15^{\circ}$ shifts, but for CCLC with varying pitch, the reflectivity peak does not shift. 

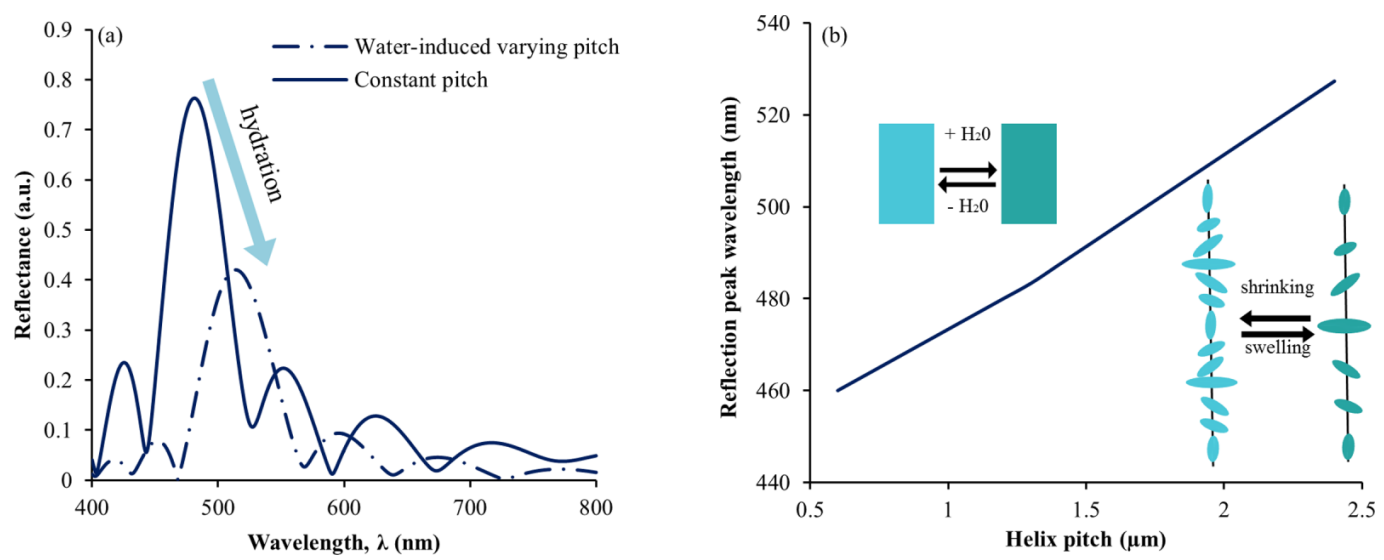

FIG. 15. Change of the reflection peaks of the CCLC free surface by humidity-driven helix pitch expansion. (a) Comparison of the FDTD simulation results for reflectivity spectrum of the surface ultrastructure in the dry and wet states under normal incidence for a reflection angle of $45^{\circ}$. The water-induced varying pitch grating structure shows broader band spectrum and less intense reflection (dashed line). The varying pitch reduces the spectrum by $44 \%$ and shifts $28 \mathrm{~nm}$ towards IR region for a reflection angle of $45^{\circ}$. (b) The linear correlation between the reflection peaks and the CCLC helix pitch. The color changes from blue at pitch equals to $0.6 \mu \mathrm{m}$ to green at pitch equals to $0.6 \mu \mathrm{m}$.

at $\theta_{\mathrm{D}}=15^{\circ}$ for the nanostructure with constant pitch. The structure with water-induced varying pitch, however, shows a red shift for all observation angles (Fig. 14(b)) and reflects a band of 460-570 $\mathrm{nm}$ of the incoming white light. For the structure with the constant pitch, the reflection increases sharply (around 50\%) from $\theta_{\mathrm{D}}=30^{\circ}$ to $\theta_{\mathrm{D}}=45^{\circ}$. However, the spectrum of the structure with water-induced varying pitch does not move towards the infrared (IR) region and undergoes less reduction in the reflected intensity, as observed by a change of the observation angle.

The reflection spectra of the surface nanostructures with constant and water-induced varying pitch are plotted in Fig. 15(a) as a function of the wavelength $\lambda$ under normal incidence for a reflection angle of $45^{\circ}$. The results show that the reflection peak of the surface nanostructure has a red shift at $\theta_{\mathrm{D}}=45^{\circ}$ driven by a tangential water gradient (from $\lambda=477$ $\mathrm{nm}$ to $\lambda=507 \mathrm{~nm}$ ). It should be noted that we assumed that the tangential water gradient would induce non-uniform swelling of the multi-layered structure along the helix axis parallel to the surface, leading to the linear expansion of the helix pitch along $\mathrm{x}$-direction.

As shown in Fig. 15(a), the water-induced varying pitch grating structure displays a broader band spectrum and less intense reflection. The varying pitch structure reduces the spectrum by $44 \%$ and shifts $28 \mathrm{~nm}$ towards IR region in comparison with the constant pitch structure for a reflection angle of $45^{\circ}$. In order to investigate the relation between the helix pitch and the reflection peak, the reflection spectra of the surface nanostructure are computed for different constant helix pitches. We assumed that the helix pitch swells from about $0.6 \mu \mathrm{m}$ in the dry state to about $2.4 \mu \mathrm{m}$ in the fully wet state. Fig. 15(b) shows an almost linear correlation between the reflection peaks and the CCLC helix pitch. The visible reflection peak shifts from $460 \mathrm{~nm}$ to $520 \mathrm{~nm}$, with a raise of the helix pitch from $0.6 \mu \mathrm{m}$ to $2.4 \mu \mathrm{m}$ through hydration. We can note that the reflection peak of the structure displays a red shift caused by an increase of the helix pitch (corresponding to higher levels of humidity), resulting in a structural color change from blue in the dry state to green in the wet state. We point out that the surface nanostructure is able to change coloration from blue in the dry state to green in the wet state through hydration. The results indicate that the reflection wavelength is directly related to the helix pitch $\mathrm{P}(\mathrm{x})$ and the structural color can be adjusted by controlling the humidity level. Fig. 16 shows the FDTD results for diffraction patterns observed in the reflection of the surface nanostructures with constant and water-induced varying pitch. The surface structure with constant pitch (dry sate) gives rise to the specular reflection whose intensity reduces by moving towards longer wavelengths. Moreover, the structure includes the first order blazed diffraction grating for angles $|\sin \theta|>0.4$ and a weaksecond order diffraction grating for angles $|\sin \theta|>0.8$. Most of the diffraction intensities
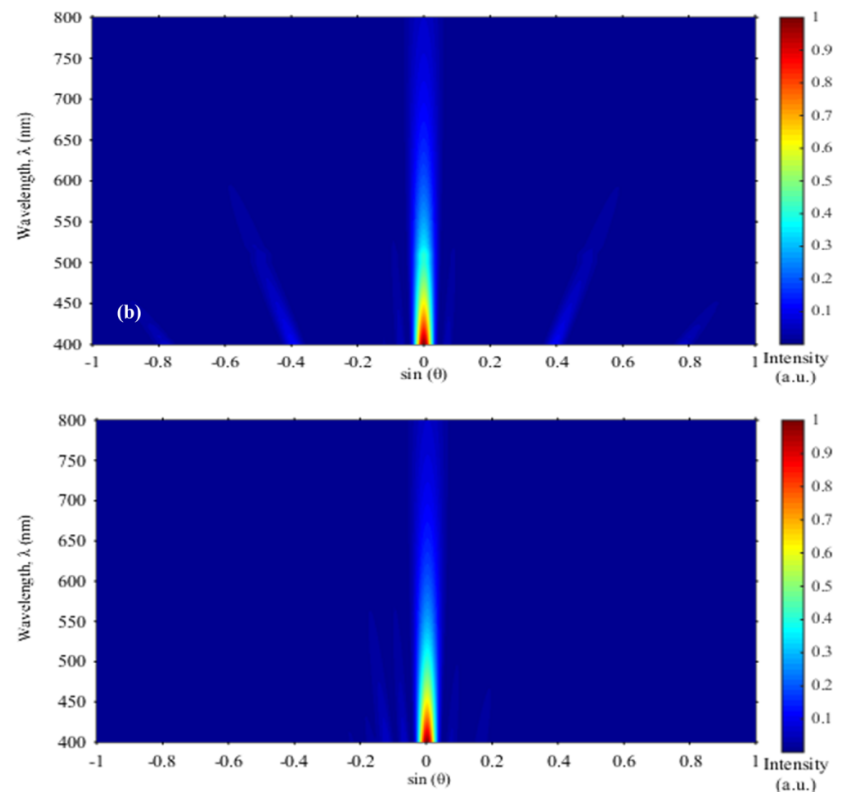

FIG. 16. FDTD simulation results for the diffraction patterns as a function of $\sin (\theta)$ and $\lambda$ observed in transmission of the surface structures with (a) constant pitch and (b) water-induced varying pitch. The central band is the signature of specular reflection. The diagonal bands in (a) are the first-order diffraction with weak second-order diffraction. 

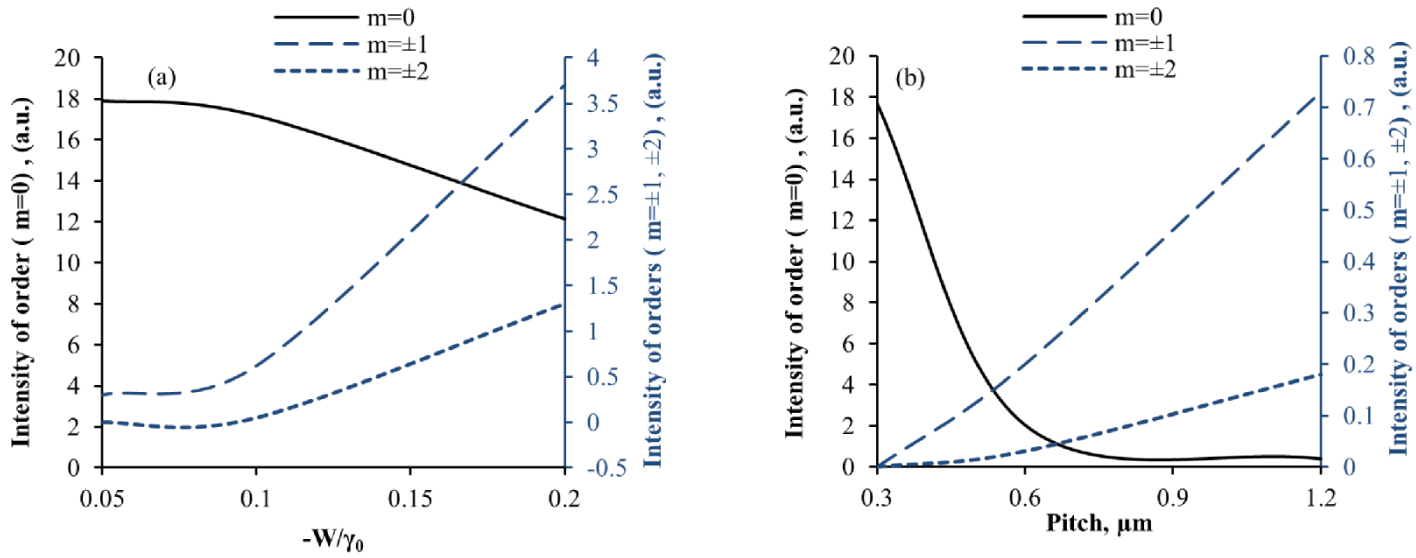

FIG. 17. Impact of the anchoring strength (a) and the helix pitch (b) on the light intensity distribution for several diffraction orders at $\lambda=490 \mathrm{~nm}$ and normal incident light for the surface nanostructures with constant pitch (dry state).

are observed at the wavelengths $\lambda<500 \mathrm{~nm}$, which is in agreement with the blue color identified for the structure at dry state (Fig. 16(a)).

The diffraction pattern detected for the structure with varying pitch (wet state) shows only the specular reflection for all wavelengths. Compared with the surface structure in the dry state, the specular reflection is broadened, a phenomenon that correlates with surface undulations with increasing periodicity. Therefore, we can infer that the iridescence occurring on the self-assembly CCLCs can be extinguished through wetting. The interplay of structure and hydration may also add to the functionality of the self-assembly CCLCs that can be used for the design of colorimetric biosensors. To quantitative investigate the role of key system parameters in the optical responses of the surface at dry and wet states, we plot in Figs. 17 and 18 the impact of the anchoring strength and the helix pitch profile on the diffracted orders. The influence of the anchoring strength on the diffracted orders for a CCLC with a fixed helix pitch $\left(\mathrm{P}_{\text {dry }}=1.2 \mu \mathrm{m}\right)$ is illustrated in Fig. 17(a). As the surface undulation amplitude grows for increasingly higher values of the anchoring strength, the intensity of specular reflection $(m=0)$ slightly decreases, and the first and second ordered diffraction gratings rise. We note that the increases of first and second ordered intensity do not significantly change with $\mathrm{W} / \gamma_{0}<0.1$. This means that for the CCLCs with higher anchoring coefficients, a structural color may be reinforced as the number of diffraction orders increases, whereas the intensity of the specular reflection $(\mathrm{m}=0)$ decreases. As shown in Fig. 17(b), similar trends are also observed through increasing the helix pitch. The results show that the decrease in the specular reflection intensity for the structures with longer pitches is very significant while the increase in first and second ordered diffraction intensities is very limited. It should be noted that the specular reflection intensity disappears for helix pitches larger than $0.9 \mu \mathrm{m}$.

The influence of the anchoring strength for a fixed waterinduced gradient pitch $\left(\mathrm{P}_{\mathrm{dry}}=1.2 \mu \mathrm{m},\left(\mathrm{P}_{\text {wet }}-\mathrm{P}_{\mathrm{dry}}\right) / \mathrm{L}=0.2\right)$ on the diffracted orders is assessed in Fig. 18(a). As the surface undulation amplitude grows for increased anchoring strengths, the intensity of specular reflection $(\mathrm{m}=0)$ noticeably diminishes in a linear fashion. A similar trend is also observed in Fig. 18(b) where the impacts of the helix pitch profile $\left(\mathrm{P}_{\mathrm{dry}}\right.$ and $\left.\left(\mathrm{P}_{\text {wet }}-\mathrm{P}_{\text {dry }}\right) / \mathrm{L}\right)$ on the distribution of the diffracted light are plotted. The results show that the decrease in specular
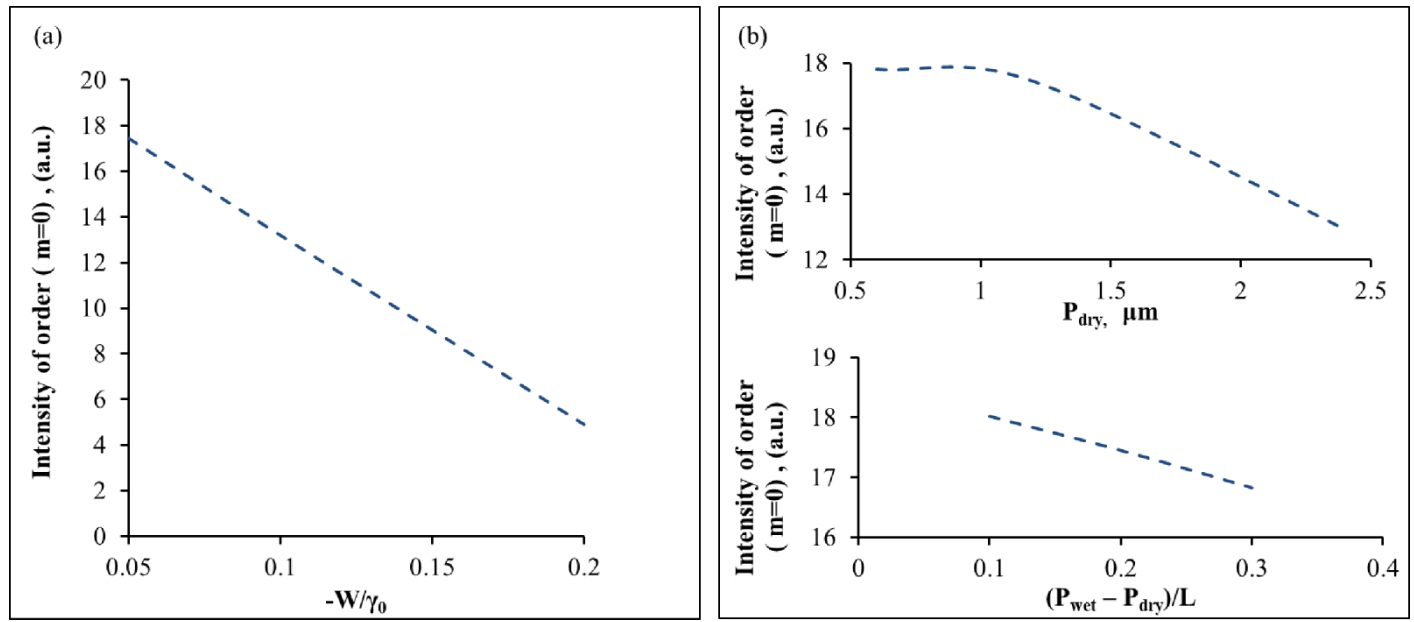

FIG. 18. Impacts of the anchoring strength (a) and the helix pitch profile (b) on the light intensity distribution $(\mathrm{m}=0)$ at $\lambda=490 \mathrm{~nm}$ and normal incident light for the surface nanostructures with water induced varying pitch (wet state). 
reflection intensity for the structures with the higher pitch gradients is very limited.

\section{CONCLUSIONS}

The formation of free surface nanostructures in cellulosic cholesteric liquid crystals with water-induced varying pitch has been investigated using a non-linear nemato-capillarity shape equation. The generalized Laplace equation based on the Cahn-Hoffman capillarity vector formalism was formulated and used as an efficient tool to analyse nano-scale surface reliefs in plant-based plywoods with water-driven varying helical pitch through interaction of anisotropic interfacial tension and chirality changes through hydration. The spatially periodic solutions to the chiral capillary equation describe surface wrinkling, where the amplitude is in the order of nanometers and the periodicity is in the order of micrometers. The role of three capillary pressure contributions (surface area change, surface area rotation, and director gradient curvature) and bulk stress jump has been characterized, and the impacts of the varying helical pitch, the elasto-capillary length scale, and the surface anchoring strength have been established.

The scaling law for the chirality-humidity driven surface wrinkling showed that the spatially varying surface profile amplitude is mostly a function of the anchoring strength and the water-induced helix pitch gradient. Finally, the optical properties of the CCLCs free surface nanostructure with waterinduced varying pitch have been investigated and compared with the CCLCs free surface nanostructure with constant pitch. The results show that the surface structure with non-uniform pitch distribution, where the pitch length changes in different regions, reflects normal incident light with a bandwidth wider than the constant pitch surface structure. The reflection peak of the surface nanostructure can be tuned through the change in the humidity level based on the plywood helix pitch expansion. We attributed the color transition to the swelling and shrinking of the multi-layered plant cell wall plywood, which cause the helicoidal pitch to increase, thereby leading to a red shift in the iridescence. In conclusion, plant-based cholesteric liquid crystals are of interest because their optical response can be controlled by self-assembly and their colorimetric humidity sensing can reduce the sensor cost. The potential biosensors can respond to different ranges of relative humidity depending on the amplitude and wavelength of the grating structure. Furthermore, as it is expected that the amount of water absorption in CCLC free surface nanostructure depends on the temperature, it is essential to consider the temperaturesensitivity of the biosensor response. All these findings provide a foundation to understand structural color phenomena in Nature and for the design of optical sensor devices.

\section{ACKNOWLEDGMENTS}

Financial support for this research was provided by Le Fonds Quebecois de la Recherche sur la Nature et les Technologies (FQRNT). P.R. is the recipient of postgraduate scholarships from McGill Engineering Doctoral Awards (MEDA). P.R. wishes to thank Professor E. Doedel for guidance with the use of the software package AUTO.
${ }^{1}$ J. E. Kirkwood and G. G. Fuller, Langmuir 25(5), 3200-3206 (2009).

${ }^{2}$ P. J. Willcox, S. P. Gido, W. Muller, and D. L. Kaplan, Macromolecules 29(15), 5106-5110 (1996).

${ }^{3}$ V. Sharma, M. Crne, J. O. Park, and M. Srinivasarao, Science 325(5939), 449-451 (2009).

${ }^{4}$ A. F. Miller and A. M. Donald, Biomacromolecules 4(3), 510-517 (2003).

${ }^{5}$ X. Y. Mu and D. G. Gray, Langmuir 30(31), 9256-9260 (2014).

${ }^{6}$ R. Meister, H. Dumoulin, M. A. Halle, and P. Pieranski, J. Phys. II 6(6), 827-844 (1996).

${ }^{7}$ P.-G.de Gennes and J. Prost, The Physics of Liquid Crystals, 2nd ed. (Clarendon Press; Oxford University Press, Oxford, New York, 1995).

${ }^{8}$ Y. Bouligand, J. Phys. 35(3), 215-236 (1974).

${ }^{9}$ Y. Bouligand, Tissue Cell 4(2), 189-217 (1972).

${ }^{10} \mathrm{~A}$. C. Neville, Biology of Fibrous Composites: Development Beyond the Cell Membrane (Cambridge University Press, New York, NY, USA, 1993).

${ }^{11}$ T. L. Tan, D. Wong, and P. Lee, Opt. Express 12(20), 4847-4854 (2004).

${ }^{12}$ A. R. Parker, Proc. R. Soc. B 262(1365), 349-355 (1995).

${ }^{13}$ A. R. Parker and N. Martini, Mater. Today: Proc. 1, 138-144 (2014).

${ }^{14}$ S. Vignolini, E. Moyroud, B. J. Glover, and U. Steiner, J. R. Soc., Interface 10(87), 20130394 (2013).

${ }^{15}$ F. Schenk, B. D. Wilts, and D. G. Stavenga, Bioinspiration \& Biomimetics 8(4), 045002 (2013).

${ }^{16}$ V. Sharma, M. Crne, J. O. Park, and M. Srinivasarao, Mater. Today: Proc. 1, 161-171 (2014).

${ }^{17}$ P. Vukusic, J. R. Sambles, and C. R. Lawrence, Nature 404(6777), 457 (2000).

${ }^{18}$ K. S. Gould and D. W. Lee, Am. J. Bot. 83(1), 45-50 (1996).

${ }^{19}$ R. M. Graham, D. W. Lee, and K. Norstog, Am. J. Bot. 80(2), 198-203 (1993).

${ }^{20}$ H. M. Whitney, M. Kolle, P. Andrew, L. Chittka, U. Steiner, and B. J. Glover, Science 323(5910), 130-133 (2009).

${ }^{21}$ H. Kasukawa, N. Oshima, and R. Fujii, Zool. Sci. 4(2), 243-257 (1987).

${ }^{22}$ N. F. Hadley, Science 203(4378), 367-369 (1979).

${ }^{23}$ H. E. Hinton and G. M. Jarman, Nature 238(5360), 160-161 (1972).

${ }^{24}$ F. Liu, B. Q. Dong, X. H. Liu, Y. M. Zheng, and J. Zi, Opt. Express 17(18), 16183-16191 (2009).

${ }^{25}$ D. W. Lee and J. B. Lowry, Nature 254(5495), 50-51 (1975).

${ }^{26}$ N. Herzer, H. Guneysu, D. J. D. Davies, D. Yildirim, A. R. Vaccaro, D. J. Broer, C. W. M. Bastiaansen, and A. P. H. J. Schenning, J. Am. Chem. Soc. 134(18), 7608-7611 (2012).

${ }^{27}$ G. Agez, R. Bitar, and M. Mitov, Soft Matter 7(6), 2841-2847 (2011).

${ }^{28}$ S. N. Fernandes, Y. Geng, S. Vignolini, B. J. Glover, A. C. Trindade, J. P. Canejo, P. L. Almeida, P. Brogueira, and M. H. Godinho, Macromol. Chem. Phys. 214(1), 25-32 (2013).

${ }^{29}$ O. V. Manyuhina, Eur. Phys. J. E 37, 48-52 (2014).

${ }^{30}$ A. D. Rey, Soft Matter 3(11), 1349-1368 (2007).

${ }^{31}$ P. Rofouie, D. Pasini, and A. D. Rey, Soft Matter 11(6), 1127-1139 (2015).

${ }^{32}$ P. Rofouie, D. Pasini, and A. D. Rey, Colloids Interface Sci. Commun. 1, 23-26 (2014).

${ }^{33}$ A. G. Cheong and A. D. Rey, J. Chem. Phys. 117(10), 5062-5071 (2002).

${ }^{34}$ A. Rapini and M. Papoular, J. Phys. Colloq. 30, C4-54 (1969).

${ }^{35}$ D. K. Hwang and A. D. Rey, Liq. Cryst. 32(4), 483-497 (2005).

${ }^{36}$ D. K. Hwang and A. D. Rey, J. Chem. Phys. 125(17), 174902 (2006).

${ }^{37}$ J. C. Roland, D. Reis, and B. Vian, Tissue Cell 24(3), 335-345 (1992).

${ }^{38} \mathrm{C}$. T. Brett and K. Waldron, Physiology and Biochemistry of Plant Cell Walls, 2nd ed. (Chapman \& Hall, London, 1996).

${ }^{39}$ Y. K. Murugesan and A. D. Rey, Polymers 2(4), 766-785 (2010).

${ }^{40}$ Y. K. Murugesan and A. D. Rey, J. Non-Newtonian Fluid Mech. 165(1-2), 32-44 (2010).

${ }^{41}$ A. D. Rey, Soft Matter 6(15), 3402-3429 (2010).

${ }^{42}$ B. I. Outram and S. J. Elston, J. Appl. Phys. 113(4), 043103 (2013).

${ }^{43}$ P. S. Salter, G. Carbone, S. A. Jewell, S. J. Elston, and P. Raynes, Phys. Rev. E 80(4), 041707 (2009).

${ }^{44}$ P. E. Cladis and M. Kleman, Mol. Cryst. Liq. Cryst. 16(1-2), 1-20 (1972).

${ }^{45}$ A. Saupe, Mol. Cryst. Liq. Cryst. 21(3-4), 211-238 (1973).

${ }^{46}$ M. Kléman and O. D. Lavrentovich, Soft Matter Physics: An Introduction (Springer, New York, 2003).

${ }^{47}$ A. P. Singh and A. D. Rey, J. Phys. II 5(9), 1321-1348 (1995).

${ }^{48}$ R. N. Thurston, J. Appl. Phys. 52(4), 3040-3052 (1981).

${ }^{49}$ A. Matsuyama, J. Chem. Phys. 139(17), 174906 (2013).

${ }^{50}$ A. Matsuyama, J. Chem. Phys. 141(18), 184903 (2014).

${ }^{51}$ L. Salmen, C. R. Biol. 327(9-10), 873-880 (2004). 
${ }^{52}$ D. J. Broer, G. N. Mol, J. A. M. M. van Haaren, and J. Lub, Adv. Mater. 11(7), 573-578 (1999).

${ }^{53}$ Z. J. Lu, L. Li, H. Vithana, Y. Q. Jiang, and S. M. Faris, Mol. Cryst. Liq. Cryst. Sci. Technol., Sect. A 301, 237-248 (1997).

${ }^{54}$ D. W. Hoffman and J. W. Cahn, Surf. Sci. 31(1), 368-388 (1972).

${ }^{55}$ A. G. Cheong and A. D. Rey, Phys. Rev. E 66(2), 021704 (2002).

${ }^{56}$ A. D. Rey, J. Chem. Phys. 120(4), 2010-2019 (2004).

${ }^{57} \mathrm{E}$. Doedel and B. Oldeman, Auto-07p: Continuation and bifurcation software for ordinary differential equations (Concordia University, 2012), http://www.dam.brown.edu/people/sandsted/auto/auto07p.pdf.
${ }^{58}$ S. Faetti and V. Palleschi, J. Phys. 46(3), 415-424 (1985).

${ }^{59}$ J. Yoshioka, F. Ito, Y. Suzuki, H. Takahashi, H. Takizawa, and Y. Tabe, Soft Matter 10(32), 5869-5877 (2014).

${ }^{60}$ N. R. Bernardino, M. C. F. Pereira, N. M. Silvestre, and M.M.T.da Gama, Soft Matter 10(47), 9399-9402 (2014).

${ }^{61}$ B. D. Terris, R. J. Twieg, C. Nguyen, G. Sigaud, and H. T. Nguyen, Europhys. Lett. 19(2), 85-90 (1992).

${ }^{62}$ M. Mitov, Adv. Mater. 24(47), 6260-6276 (2012).

${ }^{63}$ O. S. Inc., OptiFDTD technical background and tutorials—Finite difference time domain photonics simulation software, Optiwave, 2011. 